\title{
Asymmetric Hydrogenation of Ketones Using a Ruthenium(II) Catalyst Containing BINOL-Derived Monodonor Phosphorus-Donor Ligands.
}

Yingjian Xu, ${ }^{a}$ Nat W. Alcock, ${ }^{b}$ Guy J. Clarkson, ${ }^{b}$ Gordon Docherty, ${ }^{\mathrm{c}}$ Gary Woodward ${ }^{\mathrm{c}}$ and Martin Wills $^{a}$

${ }^{a}$ Asymmetric Catalysis Group, ${ }^{b}$ X-ray Crystallography Unit, Department of Chemistry, University of Warwick, Coventry, CV4 $7 A L$.

${ }^{c}$ Rhodia Consumer Specialities Limited, PO Box 80, Trinity Street, Oldbury, West Midlands, B69 4LN.

Supporting Information

\section{Contents}
A) General
B) Synthesis of (S)-BrXuPHOS
C) Synthesis of $(S)$-MeO XuPHOS, $(R)$-biPh XuPHOS , $(S)$-Me XuPHOS
and $(S)$-PhPHOS
D) Synthesis of $(S)$-ethylPHOS
E) Synthesis of $(S)$-monoPHOS
F) Synthesis of $(S)$-iso propyl phosphite
G) Synthesis of the Ru (II) complexes
$\mathrm{H})$ General experimental procedure for the asymmetric hydrogenation catalysed by $(S, S, S S)$ $\mathrm{Ru}-\mathrm{BrXuPHOS}$
I) HPLC, GC analysis and optical rotation data of the reduction products
J) References
S17
K) X-ray crystallographic data for $\mathrm{Ru}(\mathrm{II})$ complex derived from $\mathbf{8}$
L) $1 \mathrm{H}-\mathrm{NMR}$ spectra for organic compounds lacking $\mathrm{CHN}$ analysis data 


\section{A) General}

All reactions, unless otherwise stated, were run under an atmosphere of argon in flame or oven dried glassware (round bottomed flasks or Schlenk tubes). Room temperature refers to ambient room temperature $\left(20-22{ }^{\circ} \mathrm{C}\right), 0{ }^{\circ} \mathrm{C}$ refers to an ice slush bath and $-78{ }^{\circ} \mathrm{C}$ refers to a dry ice-acetone bath. Heated experiments were conducted using thermostatically controlled oil baths. Reactions were monitored by Thin layer Chromatography (TLC) using aluminium backed silica gel $60\left(\mathrm{~F}_{254}\right)$ plates, visualized using $\mathrm{UV}_{254 \mathrm{~nm}}$ and PMA, potassium permanganate or ninhydrin dips as appropriate. Flash column chromatography was carried out routinely using $60 \AA$ silica gel (Merk). NMR spectra were recorded on Bruker DPX-300 (300 MHz) or DPX-400 (400 MHz) spectrometer. Chemical shifts are reported in $\delta$ units, parts per million downfield from $\left(\mathrm{CH}_{3}\right)_{4} \mathrm{Si}$. Coupling constants $(\mathrm{J})$ are measured in hertz. IR spectra were recorded on a Nicolet Model Avatar 320 FTIR instrument. Mass spectra were recorded on a 7070E VG mass spectrometer. Enantiomeric excesses were determined by HPLC analysis (using a Merck-Hitachi L-6200 A intelligent pump, Merk-Hitachi L-4000 UV detector, Axxiom chromatography data system and controller model 727 and a Daicel Chiralcel OD 4.6 × $250 \mathrm{~mm}$ column) or GC analysis (Hewlett Packard 5890A gas chromatography, Cyclodextrin- $\beta-236 \mathrm{M}-19$ (CHROMPAC, $50 \mathrm{~m})$ ) as stated. Elemental analyses were performed using the Exeter Analytical Model CE440. Melting points were recorded on a Stuart Scientific SMP 1 instrument. Optical rotations were measured on a Perkin-Elmer 241 polarimeter. 


\section{B) Synthesis of (S)-BrXuPHOS 8}

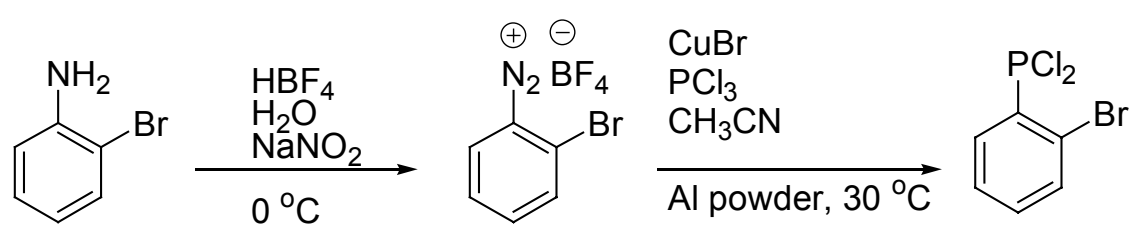<smiles>POc1ccc2ccccc2c1-c1c(OPc2ccccc2Br)ccc2ccccc12</smiles>

(S)-Br XuPHOS 8
B

$\mathrm{HNMe}_{2}$

Ether $0^{\circ} \mathrm{C}, 2 \mathrm{~h}$
(S)-BINOL

Toluene

reflux, $24 \mathrm{~h}$<smiles>CN(C)c1ccccc1Br</smiles>

C

\section{Synthesis of ortho-diazo-bromobenzene $\mathbf{A}^{1}$}

Tetra-fluoboric acid (40\% in water) $(232 \mathrm{ml})$ and distilled water $(232 \mathrm{ml})$ were charged into a three-neck flask $(1000 \mathrm{ml})$ equipped with a mechanical overhead stirrer and low temperature thermometer. The mixed solution was cooled to $0{ }^{\circ} \mathrm{C}$ and 2 bromoaniline $(100 \mathrm{~g}, 0.581 \mathrm{mols})$ was dropwise charged in to the reaction mixture over a period of 10 mins. A solution of sodium nitrite $(40 \mathrm{~g}, 0.581 \mathrm{mols})$ in $100 \mathrm{ml}$ water was added dropwise to the cooled reaction mixture, during the whole process, keeping the temperature of the mixture below $0^{\circ} \mathrm{C}$. After stirring the reaction mixture for 30 mins, the viscous mixture was filtered and washed with: tetrafluoboric acid $(5 \%, 2 \mathrm{x} 100 \mathrm{ml})$, methanol $(2 \times 100 \mathrm{ml})$ and diethyl ether $(2 \times 400 \mathrm{ml})$. The pale yellow solid was blotted dry with filter papers and then dried on a high vacuum line for a minimum of 3 days. During the drying period, the pale yellow solid became slight pink which means slightly decomposition occurred (normal). After the drying the product was obtained $(131 \mathrm{~g}, 82.6 \%)$. The experimental data is as follows: IR: $v_{\max }$ solid $/ \mathrm{cm}^{-1}=3089,2284$, 1556, 1264, 1027, 770; ${ }^{1} \mathrm{H}$ NMNR (300 MHz, DMSO) $\delta=8.84(1 \mathrm{H}, \mathrm{dd}, J 8.4 \mathrm{~Hz}, J$ $1.8 \mathrm{~Hz}, \mathrm{CH}), 8.31(1 \mathrm{H}, \mathrm{dd}, J 8.4 \mathrm{~Hz}, J 1.1 \mathrm{~Hz}, \mathrm{CH}), 8.17(1 \mathrm{H}, \mathrm{td}, J 7.5 \mathrm{~Hz}, J 1.5 \mathrm{~Hz}$, $\mathrm{CH}), 7.99(1 \mathrm{H}, \mathrm{td}, J 8.7 \mathrm{~Hz}, J 0.7 \mathrm{~Hz}, \mathrm{CH}) ;{ }^{13} \mathrm{C}$ NMR $(75 \mathrm{MHz}, \mathrm{DMSO}) 142.2(\mathrm{~s})$, $135.6(\mathrm{~s}), 135.4(\mathrm{~s}), 130.7$ (s), $124.8(\mathrm{~s}), 119.0(\mathrm{~s})$;

\section{Synthesis of ortho-dichlorophosphinobromobenzene $\mathbf{B}^{2}$}

Copper (I) bromide (1.88g, $0.0131 \mathrm{mols})$ was charged in small portions to a solution of ortho-diazo-bromobenzene $(60 \mathrm{~g}, 0.219 \mathrm{mols})$ in acetonitrile $(300 \mathrm{ml})$ at room temperature. On addition of copper (I) bromide, evolution of nitrogen was observed and the solution turned black. Phosphorus trichloride $(33.5 \mathrm{ml}, 0.372 \mathrm{mols})$ was added in one portion as the evolution of nitrogen continued. The solution was slowly warmed upto $30{ }^{\circ} \mathrm{C}$ and nitrogen evolution continued for a further 30 mins (Caution! 
Control of the temperature must be very carefully to ensure the stable release of nitrogen). The reaction was slowly heated and refluxed for $30 \mathrm{mins}$ and then allowed to cool down to room temperature. Aluminium powder (20 micron) (5.91g, $0.219 \mathrm{mols}$ ) was added and stirred overnight. After this period the reaction mixture was refluxed for $1 \mathrm{~h}$ and allowed to cool down to room temperature. Filtration of the reaction mixture through a piece of filtrate paper allowed the separation of the aluminium powder. The acetonitrile was removed by use of a high vacuum pump to leave a brown semi solid. Distillation under reduced pressure provided several lower boiling fractions with the product obtained at $97{ }^{\circ} \mathrm{C} / \mathrm{torr},(15.8 \mathrm{~g}, 28 \%)$. The experimental data was in agreement with that reported in the literature. $(21,29)$ IR: $v_{\max }$ solid $/ \mathrm{cm}^{-1}=3062,1559,1426,1100,1021,748 ;{ }^{1} \mathrm{H}$ NMR $\left(300 \mathrm{MHz}, \mathrm{CDCl}_{3}\right)$ $\delta=8.11-8.08(1 \mathrm{H}, \mathrm{dt}, J 7.98 \mathrm{~Hz}, \mathrm{Ar}-\mathrm{H}), 7.61-7.57(1 \mathrm{H}, \mathrm{m}, \mathrm{Ar}-\mathrm{H}), 7.52(1 \mathrm{H}, \mathrm{t}, J 6.83$, $\mathrm{Ar}-\mathrm{H}), 7.39(1 \mathrm{H}, \mathrm{dt}, J 6.08 \mathrm{~Hz}, \mathrm{Ar}-\mathrm{H}) ;{ }^{13} \mathrm{C} \mathrm{NMR}\left(75 \mathrm{MHz}, \mathrm{CDCl}_{3}\right) \quad \delta=133.5(\mathrm{~s})$, 132.7 (s), 131.6 (s), 128.2 (s), 126.5 (s), 125.9 (s); ${ }^{31} \mathrm{P}$ NMR (162 MHz, $\left.\mathrm{CDCl}_{3}\right) \quad \delta=$ 154.5; MS: $m / z(\mathrm{EI})=256\left([\mathrm{M}]^{+}, 78 \%\right), 223(100 \%), 177(80 \%), 107(72 \%)$; HRMS: calc for $\mathrm{C}_{6} \mathrm{H}_{4} \mathrm{BrCl}_{2} \mathrm{P}: 255.8606\left([\mathrm{M}]^{+}\right)$. Found 255.8606 .

\section{Synthesis of ortho-bis(dimethylaminophosphino)bromobenzene $\mathbf{C}^{3}$}

Ortho-dichloride-phosphino bromobenzene was charged into normal degassed diethyl ether $(400 \mathrm{ml})$ at $0{ }^{\circ} \mathrm{C}$. After stirring for 10 mins, dimethylamine gas was charged into the reaction flask through a needle above the surface of the reaction mixture. The input time of the dimethylamine gas was about 15-20 mins, and during the period, more and more white inorganic salts came out, and the solution became a suspension. After the charge of the dimethylamine gas was complete, solution was stirred in the ice bath for $2 \mathrm{~h}$. The inorganic salts were filtrated off and the resulting clear solution was evaporated under reduced pressure. The resulting oil was distilled at $106{ }^{\circ} \mathrm{C} / 0.2$ torr to obtain the final pure product which was a clear and colourless oil $(5.24 \mathrm{~g}$, $98.6 \%)$. The experimental data was in agreement with that reported in the literature. ${ }^{1} \mathrm{H}$ NMR $\left(300 \mathrm{MHz}, \mathrm{CDCl}_{3}\right) \quad \delta=7.56-7.52(1 \mathrm{H}, \mathrm{m}, \mathrm{Ar}-\mathrm{H}), 7.48-7.44(1 \mathrm{H}, \mathrm{m}, \mathrm{Ar}-\mathrm{H})$, 7.33-7.26 (1H, m, Ar-H), 7.15-7.11 (1H, m, Ar-H); ${ }^{13} \mathrm{C} \mathrm{NMR}\left(75 \mathrm{MHz}, \mathrm{CDCl}_{3}\right) \delta=$ 143.5 (s), 134.02 (s), 133.1 (s), 129.66 (s), 127.28 (s), 126.95 (s), 41.57 (d, $\mathrm{NMe}_{2}$ ); ${ }^{31} \mathrm{P}$ NMR $\left(162 \mathrm{MHz}, \mathrm{CDCl}_{3}\right) \quad \delta=101.7$. MS: $m / z(\mathrm{EI})=274\left([\mathrm{M}]^{+}, 40 \%\right), 230$ (100\%), 187 (65\%), 107 (75\%); HRMS: calc for $\mathrm{C}_{10} \mathrm{H}_{16} \mathrm{BrN}_{2} \mathrm{P}: 274.0229$ ([M] $]^{+}$). Found 274.0228.

\section{Synthesis of (S)-Br XuPHOS $\mathbf{8}^{4,5}$}

To a solution containing ortho-bis(dimethylamino)phosphinobromobenzene $(0.822 \mathrm{~g}$, $0.003 \mathrm{~mol})$ dissolved in toluene $(25 \mathrm{ml})$ was charged a solution of $(S)$-bi-2-naphthol $(0.859 \mathrm{~g}, 0.003 \mathrm{~mol})$ in Toluene $(25 \mathrm{ml})$. The reaction flask was placed in an oil bath and kept stirring at room temperature for $10 \mathrm{mins}$, and then was heated up to reflux for $24 \mathrm{~h}$. The reaction was monitored by ${ }^{31} \mathrm{P} \mathrm{NMR}$, and the released dimethylamine gas was monitored by $\mathrm{pH}$ paper. After the reaction finished, it was allowed to cool down to room temperature. Solvent was removed to get a yellow solid. $30 \mathrm{ml}$ of degassed pentane was charged into the oil above and stirred overnight. The resulting suspension was filtered and rinsed with further pentane. The off white solid was left to dry under high vacuum. The solid was purified by recrystalization with toluene to give slightly yellow crystals $(1.17 \mathrm{~g}, 82 \%)$. m.p. $225-227^{\circ} \mathrm{C} ;[\alpha]_{\mathrm{D}}{ }^{27}=+46.0(c 0.2$, $\mathrm{CH}_{2} \mathrm{Cl}_{2}$ ); IR: $v_{\max }$ solid $/ \mathrm{cm}^{-1}=3053,1226,1199,948,821,803,749 ;{ }^{1} \mathrm{H}$ NMR $(300$ 
$\left.\mathrm{MHz}, \mathrm{CDCl}_{3}\right) \delta=8.05-8.02(2 \mathrm{H}, \mathrm{m}, \mathrm{Ar}-\mathrm{H}), 7.82-7.80(1 \mathrm{H}, \mathrm{d}, \mathrm{Ar}-\mathrm{H}), 7.62-7.59(3 \mathrm{H}$, $\mathrm{m}, \mathrm{Ar}-\mathrm{H}), 7.47-7.18(8 \mathrm{H}, \mathrm{m}, \mathrm{Ar}-\mathrm{H}), 7.03-6.96(1 \mathrm{H}, \mathrm{m}, \mathrm{Ar}-\mathrm{H}), 6.82-6.78(1 \mathrm{H}, \mathrm{m}, \mathrm{Ar}-$ $\mathrm{H}) ;{ }^{13} \mathrm{C}$ NMR $\left(75 \mathrm{MHz}, \mathrm{CDCl}_{3}\right) \delta=149.99(\mathrm{~s}), 149.20(\mathrm{~s}), 133.35(\mathrm{~s}), 133.17$ (d, $J_{C P}$ $5.75 \mathrm{~Hz}), 132.8(\mathrm{~s}), 132.1(\mathrm{~s}), 131.9\left(\mathrm{~d}, J_{C P} 4.60 \mathrm{~Hz}\right), 131.1(\mathrm{~s}), 129.87(\mathrm{~s}), 128.8(\mathrm{~d}$, $\left.J_{C P} 8.62 \mathrm{~Hz}\right), 128.2(\mathrm{~s}), 127.2\left(\mathrm{~d}, J_{C P} 5.17 \mathrm{~Hz}\right), 126.7(\mathrm{~s}), 126.5(\mathrm{~s}), 125.5(\mathrm{~s}), 125.2$ (s), $121.9\left(\mathrm{~d}, J_{C P} 5.75 \mathrm{~Hz}\right) ;{ }^{31} \mathrm{P}$ NMR $\left(162 \mathrm{MHz}, \mathrm{CDCl}_{3}\right) \delta=174.7$. MS: $m / z(\mathrm{EI})=$ 471([M+H] $\left.]^{+}, 100 \%\right), 286(90 \%), 268(30 \%), 239(40 \%)$; HRMS: calc for $\mathrm{C}_{26} \mathrm{H}_{16} \mathrm{BrO}_{2} \mathrm{P}: 468.9993\left([\mathrm{M}-\mathrm{H}]^{+}\right)$. Found 468.9977.

\section{C) Synthesis of (S)-MeO XuPHOS 7, (R)-biPh XuPHOS 6, (S)-Me XuPHOS 9 and $(S)$-PhPHOS 5.}

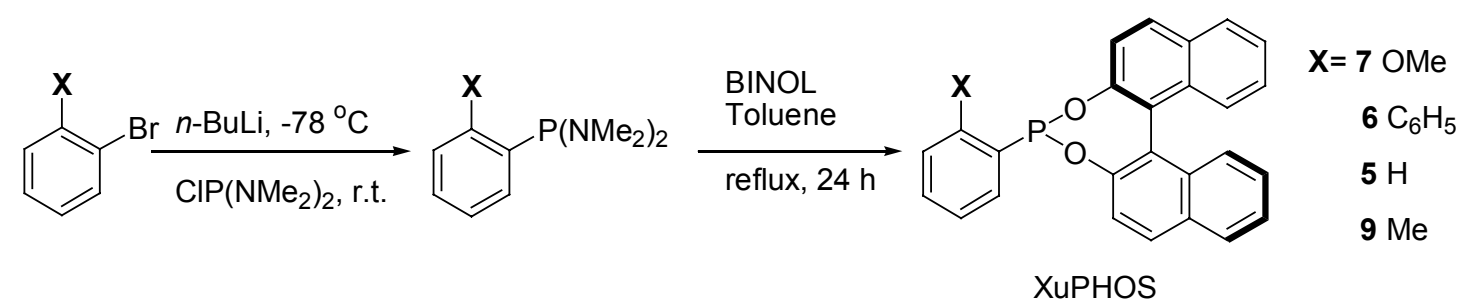

\section{Synthesis of ortho-bis(dimethylaminophosphino)anisole ${ }^{4}$}

In an oven dried $100 \mathrm{ml}$ round bottom flask twice purged with nitrogen, 2bromoanisole $(1.00 \mathrm{~g}, 5.3 \mathrm{mmol})$ was dissolved in freshly distilled diethyl ether $(25 \mathrm{ml})$ and it was allowed to cool to $-78{ }^{\circ} \mathrm{C}$. To the resulting solution was added $n$ butyllithium $(1.6 \mathrm{M}, 5.8 \mathrm{mmol}, 3.63 \mathrm{ml}, 1.1 \mathrm{eq})$ and the mixture was allowed to stir for 30 mins whilst being maintained at $-78{ }^{\circ} \mathrm{C}$. The reaction mixture was then allowed to warm to $0{ }^{\circ} \mathrm{C}$ and bis-(dimethylamino)chlorophosphane $(5.8 \mathrm{mmol}, 0.897 \mathrm{~g}, 0.85 \mathrm{ml}$, $1.1 \mathrm{eq}$ ) was added dropwise via a nitrogen purged syinge and the reaction mixture was allowed to stir 20-30 mins at ambient temperature. The resulting white suspension was washed with saturated degassed sodium hydrogen carbonate solution $(25 \mathrm{ml})$, diluted with diethyl ether $(25 \mathrm{ml})$ and transferred to a separating funnel, the organic layer was collected and the aqueous layer back extracted with a further aliquot of ether $(5 \mathrm{ml})$. The combined organic layers were dried over magnesium sulphate for half an hour, filtered and concentrated under reduced pressure to give the crude product as a pale green oil $(696 \mathrm{mg}, 58 \%)$. b.p. $95^{\circ} \mathrm{C}(0.5 \mathrm{Torr})$; IR: $v_{\max }$ neat $/ \mathrm{cm}^{-1}=$ 2859, 2252, 1583, 1466, 1236, 948; ${ }^{1} \mathrm{H}$ NMR $\left(300 \mathrm{MHz}, \mathrm{CDCl}_{3}\right) \quad \delta=7.70-7.66(1 \mathrm{H}$, $\mathrm{m}, \mathrm{Ar}-\mathrm{H}), 7.29-7.22(1 \mathrm{H}, \mathrm{m}, \mathrm{Ar}-\mathrm{H}), 7.09-7.07(1 \mathrm{H}, \mathrm{m}, \mathrm{Ar}-\mathrm{H}), 6.65(1 \mathrm{H}, \mathrm{dd}, J 4.3 \mathrm{~Hz}, J$ $8.1 \mathrm{~Hz}, \mathrm{Ar}-\mathrm{H}), 3.77\left(3 \mathrm{H}, \mathrm{s}, \mathrm{CH}_{3}\right), 2.58\left(12 \mathrm{H}, \mathrm{d}, J_{\mathrm{PH}} 18.4 \mathrm{~Hz}, 2 \mathrm{NMe}_{2}\right) ;{ }^{13} \mathrm{C}$ NMR $(75$ $\left.\mathrm{MHz}, \mathrm{CDCl}_{3}\right) \delta=161.5\left(\mathrm{~d}, J_{\mathrm{CP}} 14.9 \mathrm{~Hz}\right.$, ipso C), $133.0\left(\mathrm{~d}, J_{\mathrm{CP}} 5.7 \mathrm{~Hz}, \mathrm{Ar}-\mathrm{C}\right), 129.9$ (Ar-C), 128.7 (d, $J_{\mathrm{CP}} 11.5 \mathrm{~Hz}$, ipso C), 121.2 (Ar-C), 110.8 (Ar-C), $55.7\left(\mathrm{CH}_{3}\right), 42.0$ $\left(\mathrm{d}, J_{\mathrm{CP}} 18.4 \mathrm{~Hz}, \mathrm{~N}\left(\mathrm{CH}_{3}\right)_{2}\right) ;{ }^{31} \mathrm{P}$ NMR $\left(162 \mathrm{MHz}, \mathrm{CDCl}_{3}\right) \delta=97.7 ; \mathrm{MS}: m / z(\mathrm{EI})=$ $226\left([\mathrm{M}]^{+}, 20 \%\right), 182(100 \%), 139(70 \%)$; HRMS: calc for $\mathrm{C}_{11} \mathrm{H}_{19} \mathrm{~N}_{2} \mathrm{OP}: 226.1235$ $\left([\mathrm{M}]^{+}\right)$. Found 226.1230 . 


\section{Synthesis of $(S)-\mathrm{MeO}$ XuPHOS $7 \mathbf{a}^{4,5}$}

To a solution containing 2-bis-(dimethylaminophosphino)-anisole (500mg, $2.2 \mathrm{mmol})$ dissolved in toluene $(25 \mathrm{ml})$ was charged the $(S)$-bi-2-naphthol $(630 \mathrm{mg}, 2.2 \mathrm{mmol})$ dissolved in anhydrous toluene $(25 \mathrm{ml})$. The reaction flask was placed in an oil bath and stirred at room temperature for 10 mins. It was heated up to reflux for $24 \mathrm{hrs}$. The reaction was monitored by ${ }^{31} \mathrm{P} \mathrm{NMR}$, and also the releasing dimethylamine gas was monitored by $\mathrm{pH}$ paper. After the reaction finished, it was allowed to cool down to room temperature. Solvent was removed to get a off-white solid and dried under high vacuum. The white solid was purified by recrystalization from toluene $(604 \mathrm{mg}, 65 \%)$. m.p. $234{ }^{\circ} \mathrm{C} ;[\alpha]_{\mathrm{D}}{ }^{26}=+44.8\left(c 0.2, \mathrm{CH}_{2} \mathrm{Cl}_{2}\right)$; IR: $v_{\max }$ solid $/ \mathrm{cm}^{-1}=1588,1430,1228$, 950, 822, 754; ${ }^{1} \mathrm{H}$ NMR $\left(300 \mathrm{MHz}, \mathrm{CDCl}_{3}\right) \delta=7.96-7.93(1 \mathrm{H}, \mathrm{m}), 7.83-7.79(1 \mathrm{H}, \mathrm{m})$, $7.60(2 \mathrm{H}, \mathrm{t}, J 8.1 \mathrm{~Hz}), 7.49-7.35(4 \mathrm{H}, \mathrm{m}), 7.33-7.18(3 \mathrm{H}, \mathrm{m}), 7.13-7.09(1 \mathrm{H}, \mathrm{m}), 6.96-$ $6.85(1 \mathrm{H}, \mathrm{m}), 6.80-6.77(1 \mathrm{H}, \mathrm{m}), 6.69-6.64(1 \mathrm{H}, \mathrm{m}), 3.96\left(3 \mathrm{H}, \mathrm{s}, \mathrm{CH}_{3}\right) ;{ }^{13} \mathrm{C}$ NMR $(75$ $\left.\mathrm{MHz}, \mathrm{CDCl}_{3}\right) \delta=162.65(\mathrm{~d}, J=19.17 \mathrm{~Hz}), 152.56(\mathrm{~s}), 150.31(\mathrm{~s}), 149.25(\mathrm{~s}), 132.98$ (s), $132.80(\mathrm{~d}, J 47.28 \mathrm{~Hz}), 131.26(\mathrm{~d}, J 68.41 \mathrm{~Hz}), 130.57(\mathrm{~s}), 130.33(\mathrm{~d}, J 5.03 \mathrm{~Hz})$, 128.38 (d, $J 13.08 \mathrm{~Hz}), 126.94$ (d, $J 13.08 \mathrm{~Hz}), 126.05$ (d, $J 26.16 \mathrm{~Hz}), 124.71$ (d, $J$ $39.23 \mathrm{~Hz}), 122.02$ (s), 120.50 (s), 110.49 (s), 55.95 (s); ${ }^{31} \mathrm{P}$ NMR (162 MHz, $\mathrm{CDCl}_{3}$ ) $\delta=181 ; \mathrm{MS}: \mathrm{m} / z(\mathrm{EI})=421\left([\mathrm{M}-\mathrm{H}]^{+}, 100 \%\right), 286(87 \%)$; HRMS: calc for $\mathrm{C}_{27} \mathrm{H}_{19} \mathrm{O}_{3} \mathrm{P}$ : $421.0994\left([\mathrm{M}-\mathrm{H}]^{+}\right)$. Found 421.1003; Anal. Calculated for $\mathrm{C}_{27} \mathrm{H}_{19} \mathrm{O}_{3} \mathrm{P}: \mathrm{C}, 76.77 ; \mathrm{H}$, 4.53; P, 7.33. Found: C. 76.56; H, 4.74; P, 7.16.

\section{Synthesis of Ortho-bis-(dimethylaminophosphino)-biphenyl ${ }^{3}$}

In an oven dried $250 \mathrm{ml}$ round bottom flask twice purged with nitrogen, 2bromobiphenyl $(5.00 \mathrm{~g}, 21.4 \mathrm{mmol}, 3.698 \mathrm{ml})$ was dissolved in freshly distilled diethyl ether $(100 \mathrm{ml})$ and cooled to $-78{ }^{\circ} \mathrm{C}$. To the resulting solution was added $\mathrm{n}$ butyllithium $(2.5 \mathrm{M}, 23.6 \mathrm{mmol}, 9.44 \mathrm{ml}, 1.1 \mathrm{eq})$ and the mixture was allowed to stir for 30 mins whilst being maintained at $-78{ }^{\circ} \mathrm{C}$. The reaction mixture was then allowed to warm to $0{ }^{\circ} \mathrm{C}$ and bis-(dimethylamino)-chloro-phosphane $(23.6 \mathrm{mmol}, 3.63 \mathrm{~g}, 3.43 \mathrm{ml}$, $1.1 \mathrm{eq}$ ) was added dropwise via a nitrogen purged syinge and the reaction mixture was allowed to stir 20-30 mins at ambient temperature. The resulting white suspension was washed with saturated degassed sodium hydrogen carbonate solution $(100 \mathrm{ml})$, diluted with diethyl ether $(100 \mathrm{ml})$ and transferred to a separating funnel, the organic layer was collected and the aqueous layer back extracted with a further aliquot of ether $(20 \mathrm{ml})$. The combined organic layers were dried over magnesium sulphate, filtered and concentrated under reduced pressure to give the crude product as a pale green oil. The resulting oil was taken up in hot methanol $(50 \mathrm{ml})$, the bottom of the flask scratched with a steel needle and crystallization allowed to occur at $-20{ }^{\circ} \mathrm{C}$ in the freezer. Subsequently the crystals were filtered under nitrogen on a glass sinter, washed with small amounts of cold methanol and dried for eight hours under high vacuum in a Schlenk tube to furnish a large, colorless, crystals $(0.73 \mathrm{~g}, 25 \%)$. m.p. 38 ${ }^{\circ} \mathrm{C}$. IR: $v_{\max }$ solid $/ \mathrm{cm}^{-1}=3049,2857,2784,1441,1192,950 ;{ }^{1} \mathrm{H}$ NMR $(300 \mathrm{MHz}$, $\left.\mathrm{CDCl}_{3}\right) \delta=7.58-7.54(1 \mathrm{H}, \mathrm{m}, \mathrm{Ar}-\mathrm{H}), 7.49-7.46(2 \mathrm{H}, \mathrm{m}, 2 \mathrm{Ar}-\mathrm{H}), 7.37-7.32(4 \mathrm{H}, \mathrm{m}$, 4Ar-H), 7.27- $7.19(2 \mathrm{H}, \mathrm{m}, 2 \mathrm{Ar}-\mathrm{H}), 2.39\left(12 \mathrm{H}, \mathrm{d}, J 9.00 \mathrm{~Hz}, 2 \mathrm{~N}\left(\mathrm{CH}_{3}\right){ }_{2}\right) ;{ }^{13} \mathrm{C} \mathrm{NMR}(75$ $\left.\mathrm{MHz}, \mathrm{CDCl}_{3}\right) \delta=145.6\left(\mathrm{C}^{10}, \mathrm{~d}, J_{C P} 16.67 \mathrm{~Hz}\right), 143.6\left(\mathrm{C}^{5}, \mathrm{~s}\right), 139.2\left(\mathrm{C}^{4}, \mathrm{~d}, J_{C P} 6.32 \mathrm{~Hz}\right)$, $132.1\left(\mathrm{C}^{9}, \mathrm{~d}, J_{C P} 8.05 \mathrm{~Hz}\right), 131.6(\mathrm{Ar}-\mathrm{C}, \mathrm{s}), 128.7\left(\mathrm{C}^{8}, \mathrm{~d}, J_{C P} 5.17 \mathrm{~Hz}\right), 128.6(\mathrm{Ar}-\mathrm{C})$, 128.2 (Ar-C), 127.9 (Ar-C), 126.8 (Ar-C), 41.7 (4C, d, $J_{C P} 17.51 \mathrm{~Hz}, 2 \mathrm{NMe}_{2}$ ); ${ }^{31} \mathrm{P}$ NMR $\left(162 \mathrm{MHz}, \mathrm{CDCl}_{3}\right) \delta=100.5$; Anal. Calculated for $\mathrm{C}_{16} \mathrm{H}_{21} \mathrm{~N}_{2} \mathrm{P}: \mathrm{C}, 70.57 ; \mathrm{H}$, 7.77; N, 10.29. Found: C, 70.60; H, 7.76; N, 10.02 . 


\section{Synthesis of $(R)$-biph XuPHOS $6^{4,5}$}

To a solution containing Ortho-bis-(dimethylaminophosphino) biphenyl (134mg, $0.49 \mathrm{mmol})$ dissolved in toluene $(25 \mathrm{ml})$ was charged $(R)$-bi-2-naphthol $(859 \mathrm{mg}$, $0.003 \mathrm{~mol})$ dissolved in toluene $(25 \mathrm{ml})$. The reaction flask was placed in an oil bath and stirred at room temperature for 10 mins. It was heated up to reflux for $24 \mathrm{hrs}$. The reaction was monitored by ${ }^{31} \mathrm{P} \mathrm{NMR}$, and also the releasing dimethylamine gas was monitored by $\mathrm{pH}$ paper. After the reaction finished, it was allowed to cool down to room temperature. Solvent was removed to get a off-white solid and dried under high vacuum. The white solid was purified by recrystalisation with $1 \mathrm{ml}$ of toluene to give white crystalling solid $(158 \mathrm{mg}, 69 \%)$. The experimental data is as follows: m.p. 250$252{ }^{\circ} \mathrm{C} ;[\alpha]_{\mathrm{D}}{ }^{27}:-9.0\left(c 0.1, \mathrm{CH}_{2} \mathrm{Cl}_{2}\right)$; IR: $v_{\max } \mathrm{solid} / \mathrm{cm}^{-1}=3056,1460,1226,947,825$, 801, 749; ${ }^{1} \mathrm{H}$ NMR $\left(300 \mathrm{MHz}, \mathrm{CDCl}_{3}\right) \delta=7.96-7.87(3 \mathrm{H}, \mathrm{m}, \mathrm{Ar}-\mathrm{H}), 7.73(1 \mathrm{H}, \mathrm{d}, J$ 8.67, Ar-H), 7.65-7.62 (2H, m, Ar-H), 7.50-7.24 (13H, m, Ar-H), 7.07-7.01 (1H, m, $\mathrm{Ar}-\mathrm{H}), 6.88(1 \mathrm{H}, \mathrm{d}, J 9.00 \mathrm{~Hz}) ;{ }^{13} \mathrm{C} \mathrm{NMR}\left(75 \mathrm{MHz}, \mathrm{CDCl}_{3}\right) \delta=150.4(\mathrm{~s}), 149.0\left(\mathrm{~d}, J_{C P}\right.$ $6.32 \mathrm{~Hz}), 147.8(\mathrm{~s}), 140.2\left(\mathrm{~d}, J_{C P} 7.47 \mathrm{~Hz}\right), 133.2\left(\mathrm{~d}, J_{C P} 17.82 \mathrm{~Hz}\right), 132.0(\mathrm{~s}), 131.8(\mathrm{~d}$, $\left.J_{C P} 8.05 \mathrm{~Hz}\right), 131.5(\mathrm{~s}), 130.92(\mathrm{~s}), 130.7$ (d, $\left.J_{C P} 4.60 \mathrm{~Hz}\right), 130.5\left(\mathrm{~d}, J_{C P} 3.45 \mathrm{~Hz}\right), 129.8$ (s), 129.5 (d, $\left.J_{C P} 3.45 \mathrm{~Hz}\right), 128.6(\mathrm{~s}), 128.3(\mathrm{~s}), 127.3\left(\mathrm{~d}, J_{C P} 2.30 \mathrm{~Hz}\right), 126.6(\mathrm{~s}), 126.5$ (s), $125.2\left(\mathrm{~d}, J_{C P} 12.65 \mathrm{~Hz}\right), 122.6(\mathrm{~s}), 122.0(\mathrm{~s}) ;{ }^{31} \mathrm{P}$ NMR $(162 \mathrm{MHz}, \mathrm{CDCl} 3) \delta=$ 178.7; MS: $m / z(\mathrm{EI})=467\left([\mathrm{M}-\mathrm{H}]^{+}, 100 \%\right), 268(26 \%)$; HRMS: calc for $\mathrm{C}_{32} \mathrm{H}_{21} \mathrm{O}_{2} \mathrm{P}$ : $467.1201\left([\mathrm{M}-\mathrm{H}]^{+}\right)$. Found 467.1192.

\section{Synthesis of bisdimethylaminophenylphosphine ${ }^{3}$}

Dichlorophosphinobenzene ( $2.5 \mathrm{~g}, 0.013967 \mathrm{~mol})$ was charged into normal degassed diethyl ether $(400 \mathrm{ml})$ at $0{ }^{\circ} \mathrm{C}$. After stirring for $10 \mathrm{mins}$, dimethylamine gas was charged into the reaction flask through a needle above the surface of the reaction mixture. The input time of the dimethylamine gas was about 15-20 mins. During the period, white inorganic salts came out, and the solution became a suspension. After the charge of the dimethylamine gas was complete, solution was stirred in the ice bath for $2 \mathrm{~h}$. The inorganic salts were filtrated off and the resulting clear solution was evaporated under reduced pressure. The resulting oil was distilled at $106{ }^{\circ} \mathrm{C} / 0.2$ torr to obtain the final pure product which was a clear and colourless oil $(2.74 \mathrm{~g}, 99 \%)$. The experimental data was in agreement with that reported in the literature. IR: $v_{\max }$ neat $/ \mathrm{cm}^{-1}=2875,2828,2784,1186,952,667 ;{ }^{1} \mathrm{H}$ NMR $\left(300 \mathrm{MHz}, \mathrm{CDCl}_{3}\right) \delta=7.41-$ $7.39(2 \mathrm{H}, \mathrm{m}, \mathrm{Ar}-\mathrm{H}), 7.38-7.31(2 \mathrm{H}, \mathrm{m}, \mathrm{Ar}-\mathrm{H}), 7.26-7.21(1 \mathrm{H}, \mathrm{m}, \mathrm{Ar}-\mathrm{H}), 2.66(12 \mathrm{H}$, $\left.\mathrm{d}, J 9.15 \mathrm{~Hz}, 2 \mathrm{~N}\left(\mathrm{CH}_{3}\right)_{2}\right) ;{ }^{13} \mathrm{C} \mathrm{NMR}\left(75 \mathrm{MHz}, \mathrm{CDCl}_{3}\right) \delta=141.2(\mathrm{~d}, J 4.02 \mathrm{~Hz}, \mathrm{Ar}-\mathrm{C})$, 132.6 (d, J 9.20 Hz, Ar-C), 131.6 (s, Ar-C), 131.4 (s, Ar-C), 128.7 (d, J 2.87 Hz, ArC), $127.9(\mathrm{~s}, \mathrm{Ar}-\mathrm{C}), 42.2\left(\mathrm{~d}, J 14.9 \mathrm{~Hz}, \mathrm{NMe}_{2}\right) ;{ }^{31} \mathrm{P}$ NMR $\left(162 \mathrm{MHz}, \mathrm{CDCl}_{3}\right) \delta=$ 100.25 .

\section{Synthesis of $(S)$-phPHOS $6^{4,5}$}

To a solution containing bis(dimethylamino)phosphino benzene $(1.00 \mathrm{~g}, 5.096 \mathrm{mmol})$ dissolved in toluene $(25 \mathrm{ml})$ was charged a solution of $(S)$ - bi-2-naphthol $(1.459 \mathrm{~g}$, $5.096 \mathrm{mmol})$ in Toluene $(25 \mathrm{ml})$. The reaction flask was placed in an oil bath and kept stirring at room temperature for 10 mins, and then was heated up to reflux for $24 \mathrm{~h}$. The reaction was monitored by ${ }^{31} \mathrm{P} \mathrm{NMR}$, and the released dimethylamine gas was monitored by $\mathrm{pH}$ paper. After the reaction finished, it was allowed to cool down to room temperature. Solvent was removed to get a yellow oil. $30 \mathrm{ml}$ of degassed 
pentane was charged into the oil above and stirred overnight. The resulting suspension was filtered and rinsed with further pentane. The off white solid was left to dry under high vacuum. The solid was purified by recrystalization with toluene to give slightly yellow crystals $(1.78 \mathrm{~g}, 89 \%)$. m.p. $165^{\circ} \mathrm{C}$; [ a $]_{\mathrm{D}}{ }^{19}=+180.3\left(c 0.2, \mathrm{CH}_{2} \mathrm{Cl}_{2}\right)$; IR: $v_{\max }$ solid $/ \mathrm{cm}^{-1}=3054,1587,1228,948,819,743 ;{ }^{1} \mathrm{H}$ NMR $\left(300 \mathrm{MHz}, \mathrm{CDCl}_{3}\right) \delta=8.03$ $(1 \mathrm{H}, \mathrm{d}, J 8.85 \mathrm{~Hz}, \mathrm{Ar}-\mathrm{H}), 7.95(1 \mathrm{H}, \mathrm{d}, J 8.28 \mathrm{~Hz}, \mathrm{Ar}-\mathrm{H}), 7.85(1 \mathrm{H}, \mathrm{d}, J 8.85 \mathrm{~Hz}, \mathrm{Ar}-$ H), $7.66(1 \mathrm{H}, \mathrm{d}, J 8.85 \mathrm{~Hz}, \mathrm{Ar}-\mathrm{H}), 7.59(1 \mathrm{H}, \mathrm{d}, J 8.67 \mathrm{~Hz}, \mathrm{Ar}-\mathrm{H}), 7.48-7.38(6 \mathrm{H}, \mathrm{m}$, Ar-H), 7.31- $7.23(4 \mathrm{H}, \mathrm{m}, \mathrm{Ar}-\mathrm{H}), 7.17(1 \mathrm{H}, \mathrm{d}, J 7.53 \mathrm{~Hz}, \mathrm{Ar}-\mathrm{H}), 6.70(1 \mathrm{H}, \mathrm{d}, J 8.64$ $\mathrm{Hz}, \mathrm{Ar}-\mathrm{H}) ;{ }^{13} \mathrm{C} \mathrm{NMR}\left(75 \mathrm{MHz}, \mathrm{CDCl}_{3}\right) \delta=150.4(\mathrm{~s}), 149.0(\mathrm{~d}, J 5.75 \mathrm{~Hz}), 139.3(\mathrm{~d}$, $J 37.80 \mathrm{~Hz}), 133.2(\mathrm{~d}, J 24.7 \mathrm{~Hz}), 132.4(\mathrm{~s}), 131.8(\mathrm{~d}, J 46.0 \mathrm{~Hz}), 131.1(\mathrm{~d}, J 2.88 \mathrm{~Hz})$, 130.7 (s), 129.8 (s), 128.8 (d, J 7.47 Hz), 128.5 (d, J 6.90 Hz), 127.3 (d, J 3.45 Hz), 126.6 (d, $J 18.40 \mathrm{~Hz}), 125.3$ (d, $J 17.82 \mathrm{~Hz}), 122.7$ (s), $121.9(\mathrm{~s}) ;{ }^{31} \mathrm{P}$ NMR (162 MHz, $\left.\mathrm{CDCl}_{3}\right) \delta=185.0$.

\section{Synthesis of ortho-bis(dimethylaminophosphino) toluene ${ }^{3}$}

In an oven dried $100 \mathrm{ml}$ round bottom flask twice purged with nitrogen, 2bromotoluene $(5.00 \mathrm{~g}, 29.2 \mathrm{mmol})$ was dissolved in freshly distilled diethyl ether $(125 \mathrm{ml})$ and it was allowed to cool to $-78{ }^{\circ} \mathrm{C}$. To the resulting solution was added $t$ butyllithium (1.7M, $59.9 \mathrm{mmol}, 35 \mathrm{ml}, 2.05 \mathrm{eq})$ and the mixture was allowed to stir for 30 mins whilst being maintained at $-78^{\circ} \mathrm{C}$. The reaction mixture was then allowed to warm to $0{ }^{\circ} \mathrm{C}$ and bis-(dimethylamino)chlorophosphane $(29.2 \mathrm{mmol}, 4.51 \mathrm{~g}, 4.27$ $\mathrm{ml}, 1.0 \mathrm{eq}$ ) was added dropwise via a nitrogen purged syinge and the reaction mixture was allowed to stir 20-30 mins at ambient temperature. The resulting white suspension was washed with saturated degassed sodium hydrogen carbonate solution $(125 \mathrm{ml})$, diluted with diethyl ether $(125 \mathrm{ml})$ and transferred to a separating funnel, the organic layer was collected and the aqueous layer back extracted with a further aliquot of ether $(5 \mathrm{ml})$. The combined organic layers were dried over magnesium sulphate for half an hour, filtered and concentrated under reduced pressure to give the crude product as a colourless oil (696mg, 58\%). b.p. $72.5-74.0{ }^{\circ} \mathrm{C}$ (0.05 Torr); IR: $v_{\max }$ neat $/ \mathrm{cm}^{-1}=3052,2858,1445,1189,952,665 ;{ }^{1} \mathrm{H}$ NMR $\left(300 \mathrm{MHz}, \mathrm{CDCl}_{3}\right) \delta=$ 6.90-6.86 $(1 \mathrm{H}, \mathrm{m}, \mathrm{Ar}-\mathrm{H}), 6.69-6.57(3 \mathrm{H}, \mathrm{m}, \mathrm{Ar}-\mathrm{H}), 2.17\left(12 \mathrm{H}, \mathrm{d}, J_{\mathrm{PH}} 9.06 \mathrm{~Hz}\right.$, $\left.2 \mathrm{NMe}_{2}\right), 1.74\left(3 \mathrm{H}, \mathrm{s}, \mathrm{ArCH}_{3}\right) ;{ }^{13} \mathrm{C} \mathrm{NMR}\left(75 \mathrm{MHz}, \mathrm{CDCl}_{3}\right) \delta=140.2\left(\mathrm{~d}, J_{\mathrm{CP}}\right.$ $21.84 \mathrm{~Hz}, \mathrm{Ar}-\mathrm{C}), 138.6$ (d, $\left.J_{\mathrm{CP}} 2.88 \mathrm{~Hz}, \mathrm{Ar}-\mathrm{C}\right), 130.65$ (d, $\left.J 2.88 \mathrm{~Hz}, \mathrm{Ar}-\mathrm{C}\right), 130.4(\mathrm{~d}$, $\left.J_{\mathrm{CP}} 6.90 \mathrm{~Hz}, \mathrm{Ar}-\mathrm{C}\right), 127.7$ (s, Ar-C), $125.2(\mathrm{~s}, \mathrm{Ar}-\mathrm{C}), 41.2\left(\mathrm{~d}, J 16.09 \mathrm{~Hz}, \mathrm{~N}\left(\mathrm{CH}_{3}\right)_{2}\right)$, $20.3\left(\mathrm{~d}, J_{\mathrm{CP}} 12.64 \mathrm{~Hz}, \mathrm{ArCH}_{3}\right) ;{ }^{31} \mathrm{P} \mathrm{NMR}\left(162 \mathrm{MHz}, \mathrm{CDCl}_{3}\right) \delta=98.3$; Anal. Calculated for $\mathrm{C}_{11} \mathrm{H}_{19} \mathrm{~N}_{2} \mathrm{P}$ : C, 62.84; H, 9.11; N, 13.32. Found: C, 62.87; H, 9.13; N, 13.12; P, 14.63.

\section{Synthesis of $(S)$-Me XuPHOS $9^{4,5}$}

To a solution containing 2-bis-(dimethylaminophosphino)toluene $(734 \mathrm{mg}, 3.49 \mathrm{mmol})$ dissolved in toluene $(25 \mathrm{ml})$ was charged the $(S)$-bi-2-naphthol $(1 \mathrm{~g}, 3.49 \mathrm{mmol})$ dissolved in anhydrous toluene $(25 \mathrm{ml})$. The reaction flask was placed in an oil bath and stirred at room temperature for 10 mins. It was heated up to reflux for $24 \mathrm{hrs}$. The reaction was monitored by ${ }^{31} \mathrm{P}$ NMR, and also the releasing dimethylamine gas was monitored by $\mathrm{pH}$ paper. After the reaction finished, it was allowed to cool down to room temperature. Solvent was removed to get a off-white solid and dried under high vacuum. The white solid was purified by recrystalization from toluene $(1.20 \mathrm{~g}, 85 \%)$. m.p. $186{ }^{\circ} \mathrm{C} ;[\alpha]_{\mathrm{D}}{ }^{25}=+89.5\left(c 0.2, \mathrm{CH}_{2} \mathrm{Cl}_{2}\right)$; IR: $v_{\max }$ solid $/ \mathrm{cm}^{-1}=3048,1588,1231$, 
949, 816, 746; ${ }^{1} \mathrm{H}$ NMR $\left(300 \mathrm{MHz}, \mathrm{CDCl}_{3}\right) \delta=8.03(1 \mathrm{H}, \mathrm{d}, J 8.85 \mathrm{~Hz}, \mathrm{Ar}-\mathrm{H}), 7.95$ $(1 \mathrm{H}, \mathrm{d}, J 8.10 \mathrm{~Hz}, \mathrm{Ar}-\mathrm{H}) 7.82(1 \mathrm{H}, \mathrm{d}, J 8.64 \mathrm{~Hz}, \mathrm{Ar}-\mathrm{H}), 7.63-7.58(2 \mathrm{H}, \mathrm{m}, \mathrm{Ar}-\mathrm{H}), 7.47-$ $7.36(4 \mathrm{H}, \mathrm{m}, \mathrm{Ar}-\mathrm{H}), 7.32-7.14(5 \mathrm{H}, \mathrm{m}, \mathrm{Ar}-\mathrm{H}), 6.89(1 \mathrm{H}, \mathrm{t}, J 6.96 \mathrm{~Hz}, \mathrm{Ar}-\mathrm{H}), 6.68(1 \mathrm{H}$, $\mathrm{d}, J 8.85 \mathrm{~Hz}, \mathrm{Ar}-\mathrm{H}), 2.79\left(3 \mathrm{H}, \mathrm{d}, J 2.07 \mathrm{~Hz}, \mathrm{Ar}-\mathrm{CH}_{3}\right) ;{ }^{13} \mathrm{C} \mathrm{NMR}\left(75 \mathrm{MHz}, \mathrm{CDCl}_{3}\right) \delta=$ 149.8 (d, $J$ 94.27Hz, Ar-C), 142.2 (d, J 29.89Hz, Ar-C), 133.2 (d, $J 32.2 \mathrm{~Hz}, A r-C)$, 131.72 (s, Ar-C), 131.70 (d, J 48.86Hz, Ar-C), 131.0 (s, Ar-C), 130.9 (d, J 3.45Hz, Ar-C), 129.7 (d, J 5.18Hz, Ar-C), 128.8 (d, J 8.63Hz, Ar-C), 127.3 (d, J 9.20Hz, ArC), 126.5 (d, J 20.12Hz, Ar-C), 125.7 (s, Ar-C), 125.2 (d, J 22.42Hz, Ar-C), 122.33 (s, Ar-C), 122.0 (s, Ar-C), 20.2 (d, J 23.56Hz, Ar-CH 3 ); ${ }^{31} \mathrm{P}$ NMR $\left(162 \mathrm{MHz}, \mathrm{CDCl}_{3}\right)$ $\delta=183.0$; MS: $m / z(E I)=406\left([M]^{+}, 100 \%\right), 239(32 \%)$; HRMS: calc for $\mathrm{C}_{27} \mathrm{H}_{19} \mathrm{O}_{2} \mathrm{P}$ : $406.1123\left([\mathrm{M}]^{+}\right)$. Found 406.1112.

\section{D) Synthesis of $(S)$-ethylPHOS 4}

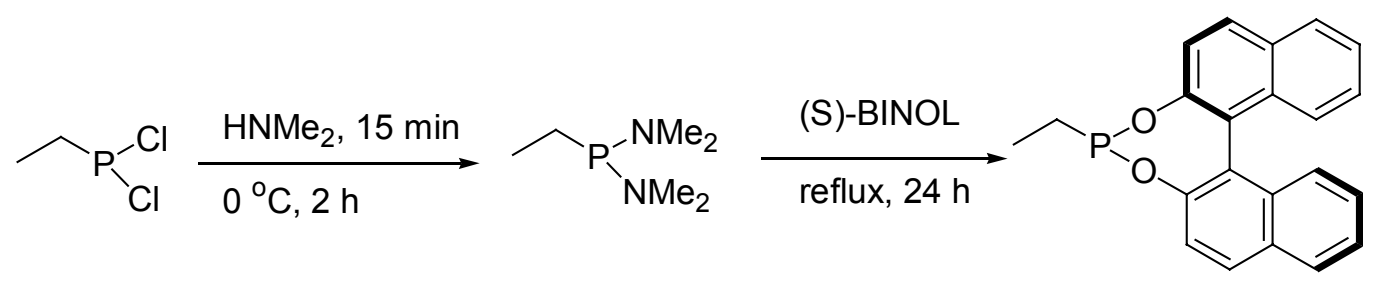

1. Synthesis of bis-dimethylaminophosphino ethane ${ }^{3}$

Dichlorophosphino ethane $(2.0 \mathrm{~g}, 1.59 \mathrm{ml}, 0.0153 \mathrm{~mol})$ was charged into normal degassed diethyl ether $(120 \mathrm{ml})$ at $0{ }^{\circ} \mathrm{C}$. After stirring for $10 \mathrm{~min}$, dimethyl amine gas was charged into the reaction flask through a needle above the surface of the reaction mixture. The input time of the dimethylamine gas was about 15-20 mins, and during the period, more and more white inorganic salts came out, and the solution became a white suspension. After the charge of the dimethylamine gas was complete, solution was stirred in the ice bath for $2 \mathrm{~h}-2.5 \mathrm{~h}$. The white inorganic salt was filtrated off under magnesium sulfate powder under argon and the resulting clear solution was evaporated under reduced pressure. The resulting clear oil was the final product $(2.24 \mathrm{~g}, 99 \%)$. IR: $v_{\max }$ neat $/ \mathrm{cm}^{-1}=2962,2876,2783,1260,949,754$; The experimental data was in agreement with that reported in the literature. ${ }^{1} \mathrm{H}$ NMR (400 $\left.\mathrm{MHz}, \mathrm{CDCl}_{3}\right) \delta=2.56\left(12 \mathrm{H}, \mathrm{dd}, J 4.56 \mathrm{~Hz}, J 9.11 \mathrm{~Hz}, \mathrm{~N}\left(\mathrm{CH}_{3}\right)_{2}\right), 1.52(2 \mathrm{H}, \mathrm{dq}, J 7.72$ $\left.\mathrm{Hz}, J 1.89 \mathrm{~Hz}, \mathrm{CH}_{2}\right), 0.88\left(3 \mathrm{H}, \mathrm{dt}, J 17.71 \mathrm{~Hz}, J 7.54 \mathrm{~Hz}, \mathrm{CH}_{3}\right) ;{ }^{13} \mathrm{C} \mathrm{NMR}(100 \mathrm{MHz}$, $\left.\mathrm{CDCl}_{3}\right) \delta=40.45\left(\mathrm{~d}, J 12.3 \mathrm{~Hz}, \mathrm{NMe}_{2}\right), 19.26\left(\mathrm{~d}, J 0.9 \mathrm{~Hz}, \mathrm{CH}_{2}\right), 8.13(\mathrm{~d}, J$ 21.4, $\left.\mathrm{CH}_{3}\right) ;{ }^{31} \mathrm{P}$ NMR $\left(162 \mathrm{MHz}, \mathrm{CDCl}_{3}\right) \delta=97.3$;

\section{Synthesis of $(S)$-ethylPHOS $4^{4,5}$}

To a solution containing bis-dimethylaminophosphino ethane $(1.00 \mathrm{~g}, 0.0067 \mathrm{~mol})$ dissolved in anhydrous toluene $(25 \mathrm{ml})$ was charged the $(S)$-bi-2-naphthol $(1.92 \mathrm{~g}$, $0.0067 \mathrm{~mol})$ dissolved in anhydrous toluene $(25 \mathrm{ml})$. The reaction flask was placed under argon in an oil bath and stirred at room temperature for $10 \mathrm{~min}$. It was heated up to reflux for $24 \mathrm{hrs}$. The reaction was monitored by ${ }^{31} \mathrm{P} \mathrm{NMR}$, and also the releasing dimethyl amine gas was monitored by wet $\mathrm{pH}$ paper. After the reaction finished, it was allowed to cool down to room temperature. Solvent was removed to get a yellow solid and dried under high vacuum. The yellow solid was purified by washing with cold $\mathrm{Et}_{2} \mathrm{O}$ to give light yellow solid $(1.95 \mathrm{~g}, 85 \%)$. m.p. $200{ }^{\circ} \mathrm{C} ;[\alpha]_{\mathrm{D}}{ }^{18}=$ 
+506.6 ( $c$ 0.1, $\mathrm{CH}_{2} \mathrm{Cl}_{2}$ ); IR: $v_{\max }$ solid $/ \mathrm{cm}^{-1}=3058,2959,1229,952,820,748$; Anal. Calculated for $\mathrm{C}_{22} \mathrm{H}_{17} \mathrm{O}_{2} \mathrm{P}: \mathrm{C}, 76.74 ; \mathrm{H}, 4.98$; P, 9.00; Found: C, 76.39; H, 4.99; P, 8.84; ${ }^{1} \mathrm{H}$ NMR $\left(400 \mathrm{MHz}, \mathrm{CDCl}_{3}\right) \delta=7.88(1 \mathrm{H}, \mathrm{d}, J 8.83 \mathrm{~Hz}, \mathrm{Ar}-\mathrm{H}), 7.81(3 \mathrm{H}, \mathrm{dd}, J$ $4.28 \mathrm{~Hz}, J 8.83 \mathrm{~Hz}, \mathrm{Ar}-\mathrm{H}), 7.41(1 \mathrm{H}, \mathrm{d}, J 8.83 \mathrm{~Hz}, \mathrm{Ar}-\mathrm{H}), 7.31(2 \mathrm{H}, \mathrm{td}, J 1.24 \mathrm{~Hz}, J$ $6.72 \mathrm{~Hz}, \mathrm{Ar}-\mathrm{H}), 7.25(3 \mathrm{H}, \mathrm{t}, J 8.19 \mathrm{~Hz}, \mathrm{Ar}-\mathrm{H}), 7.15(2 \mathrm{H}, \mathrm{t}, J 6.68 \mathrm{~Hz}$, At-H), 1.53 $\left(2 \mathrm{H}, \mathrm{m}, \mathrm{CH}_{2}\right), 1.08\left(3 \mathrm{H}, \mathrm{m}, \mathrm{CH}_{3}\right) ;{ }^{13} \mathrm{C} \mathrm{NMR}\left(100 \mathrm{MHz}, \mathrm{CDCl}_{3}\right) \delta=150.28(\mathrm{~d}, J$ 3.45Hz, Ar-C), 148.34 (d, J 6.90Hz, Ar-C), 132.72 (s, Ar-C), 131.08 (d, J 33.91Hz, Ar-C), 129.96 (d, J 71.14Hz, Ar-C), 128.15 (d, J 9.19Hz, Ar-C), 126.72 (d, J 5.75Hz, Ar-C), 126.05 (s, Ar-C), 124.68 (d, J 2.88Hz, Ar-C), 121.57 (d, J 59.20Hz, Ar-C), $26.53\left(\mathrm{~d}, J 32.18 \mathrm{~Hz}, \mathrm{CH}_{2}\right), 5.09$ (d, $\left.J 18.97 \mathrm{~Hz}, \mathrm{CH}_{3}\right) ;{ }^{31} \mathrm{P}$ NMR $\left(162 \mathrm{MHz}, \mathrm{CDCl}_{3}\right)$ $\delta=215.7 ; \mathrm{MS}: m / z(\mathrm{EI})=343\left([\mathrm{M}-\mathrm{H}]^{+}, 100 \%\right), 212(25 \%)$; HRMS: calc for $\mathrm{C}_{22} \mathrm{H}_{17} \mathrm{O}_{2} \mathrm{P}: 343.0888\left([\mathrm{M}-\mathrm{H}]^{+}\right)$. Found: 343.0887.

\section{E) Synthesis of $(S)$-monoPHOS $2^{6}$}

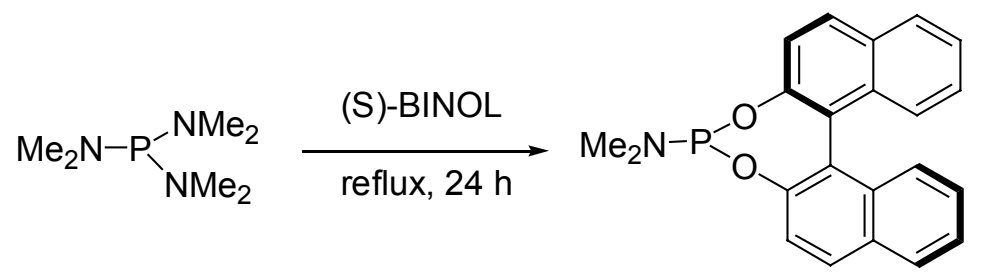

To a solution containing tris-(dimethylaminophosphino)phosphine $(0.360 \mathrm{~g}, 0.4 \mathrm{ml}$ $0.00222 \mathrm{~mol})$ dissolved in anhydrous toluene $(20 \mathrm{ml})$ was charged the $(S)$-bi-2naphthol $(0.500 \mathrm{~g}, 0.00175 \mathrm{~mol})$ dissolved in anhydrous toluene $(20 \mathrm{ml})$. The reaction flask was placed under argon in an oil bath and stirred at room temperature for 10 mins. It was heated up to reflux for $12 \mathrm{hrs}$. The reaction was monitored by ${ }^{31} \mathrm{P} \mathrm{NMR}$, and also the releasing dimethylamine gas was monitored by wet $\mathrm{pH}$ paper. After the reaction finished, it was allowed to cool down to room temperature. Solvent was removed under reduced pressure to give a yellow solid and dried under high vacuum. The resulting dry solid was purified to give a light yellow solid $(424 \mathrm{mg}, 68 \%) \mathrm{mp}$ $208-210{ }^{\circ} \mathrm{C}$; The following data is in agreement with published data. $[\alpha]_{\mathrm{D}}{ }^{22}=+683.7$ (c $0.03, \mathrm{CH}_{2} \mathrm{Cl}_{2}$ ); IR: $v_{\max }$ solid $/ \mathrm{cm}^{-1}=2775,1229,944,821,751,686 ;{ }^{1} \mathrm{H}$ NMR $(300$ $\left.\mathrm{MHz}, \mathrm{CDCl}_{3}\right) \delta=7.88(1 \mathrm{H}, \mathrm{d}, J 8.60 \mathrm{~Hz}, \mathrm{Ar}-\mathrm{H}), 7.84-7.80(3 \mathrm{H}, \mathrm{m}, \mathrm{Ar}-\mathrm{H}), 7.42(1 \mathrm{H}$, $\mathrm{d}, J 8.64 \mathrm{~Hz}, \mathrm{Ar}-\mathrm{H}), 7.37-7.24(5 \mathrm{H}, \mathrm{m}, \mathrm{Ar}-\mathrm{H}), 7.22-7.12(2 \mathrm{H}, \mathrm{m}, \mathrm{Ar}-\mathrm{H}), 2.46(6 \mathrm{H}, \mathrm{d}, J$ $\left.9.06 \mathrm{~Hz}, \mathrm{Ar}-\mathrm{CH}_{3}\right) ;{ }^{13} \mathrm{C} \mathrm{NMR}\left(75 \mathrm{MHz}, \mathrm{CDCl}_{3}\right.$ ) $\delta=131.12$ (Ar-C), 130.66 (Ar-C), 130.40 (Ar-C), 128.73 (Ar-C), 128.65 (Ar-C), 127.39 (Ar-C), 127.31 (Ar-C), 126.48 (Ar-C), 125.17 (Ar-C), 124.98 (Ar-C), 122.49 (Ar-C), 122.36 (Ar-C), 36.36 (d, $\left.\mathrm{NMe}_{2}\right) ;{ }^{31} \mathrm{P}$ NMR $\left(121 \mathrm{MHz}, \mathrm{CDCl}_{3}\right) \delta=149.9$; MS: $m / z(\mathrm{EI})=359\left([\mathrm{M}]^{+}, 100 \%\right)$, 313 (50\%); HRMS: calc for $\mathrm{C}_{22} \mathrm{H}_{18} \mathrm{NO}_{2} \mathrm{P}: 359.1075\left([\mathrm{M}]^{+}\right)$. Found 359.1077. 
F) Synthesis of (S)-iso propyl phosphite $3^{7,8}$<smiles>CCOc1ccc2ccccc2c1-c1c(OP(Oc2ccc3ccccc3c2)OC(C)C)ccc2ccccc12</smiles>

Trichloro phosphine $\mathrm{PCl}_{3}(575 \mathrm{mg}, 0.36 \mathrm{ml}, 4.19 \mathrm{mmol}, 1.2 \mathrm{e} . \mathrm{q}$.$) was dissolved in$ anhydrous toluene $(50 \mathrm{ml})$, and the solution was cooled to $-78{ }^{\circ} \mathrm{C} . \mathrm{Et}_{3} \mathrm{~N}(2.43 \mathrm{ml}$, $1.77 \mathrm{~g}, 17.45$ e.q.) was added slowly, and the reaction mixture was stirred for $5 \mathrm{~min}$. (S)-BINOL (1.0g, 3.49mmol, 1.0e.q.) was added in. The reaction mixture was stirred at $-78{ }^{\circ} \mathrm{C}$ for $90 \mathrm{~min}$, and then stirred at room temperature for $3 \mathrm{~h}$. Reaction was monitored by P31 NMR until it indicated no presence of starting material. Isopropanol $\left(210 \mathrm{mg}, 0.27 \mathrm{ml}, 3.49 \mathrm{mmol}, 1.0\right.$ e.q.) and $\mathrm{Et}_{3} \mathrm{~N}(1.22 \mathrm{ml}, 833 \mathrm{mg}, 8.73 \mathrm{mmol}$, 2.5e.q.) were added slowly. The reaction mixture was stirred for $18 \mathrm{~h}$ until P31 NMR indicated no starting material left. The crude product was purified after column and gave the final product as a white powder $(686 \mathrm{mg}, 52.5 \%) . \mathrm{mp} 80-82{ }^{\circ} \mathrm{C} .[\alpha]_{\mathrm{D}}{ }^{18}=$ +254.7 (c 0.1, $\left.\mathrm{CH}_{2} \mathrm{Cl}_{2}\right)$; IR: $v_{\max }$ solid $/ \mathrm{cm}^{-1}=3054,2967,1588,1212,938,746 ;{ }^{1} \mathrm{H}$ NMR $\left(400 \mathrm{MHz}, \mathrm{CDCl}_{3}\right) \delta=7.97-7.89(4 \mathrm{H}, \mathrm{m}, \mathrm{Ar}-\mathrm{H}), 7.51(1 \mathrm{H}, \mathrm{d}, J 8.64 \mathrm{~Hz}, \mathrm{Ar}-\mathrm{H})$, 7.44-7.34 (5H, m, Ar-H), $7.24(2 \mathrm{H}, \mathrm{t}, J 7.65 \mathrm{~Hz}, \mathrm{Ar}-\mathrm{H}), 4.58-4.47(1 \mathrm{H}, \mathrm{m}, \mathrm{CH}), 1.31$ $\left(6 \mathrm{H}, \mathrm{dd}, J 6.21 \mathrm{~Hz}, J 12.82 \mathrm{~Hz}, 2 \mathrm{CH}_{3}\right) ;{ }^{13} \mathrm{C} \mathrm{NMR}\left(100 \mathrm{MHz}, \mathrm{CDCl}_{3}\right) \delta=148.72(\mathrm{~s}, \mathrm{Ar}-$ C), $148.10(\mathrm{~s}, \mathrm{Ar}-\mathrm{C}), 133.19$ (d, $J 10.35 \mathrm{~Hz}, \mathrm{Ar}-\mathrm{C}), 131.69$ (d, $J 34.49 \mathrm{~Hz}, \mathrm{Ar}-\mathrm{C})$, 130.42 (d, J 43.69Hz, Ar-C), 128.72 (d, J 6.90Hz, Ar-C), 127.45 (s, Ar-C), 126.56 (d, $J 9.20 \mathrm{~Hz}$, Ar-C), 125.31 (d, $J 14.37 \mathrm{~Hz}, \mathrm{Ar}-\mathrm{C}), 122.26$ (d, $J 9.77 \mathrm{~Hz}, \mathrm{Ar}-\mathrm{C}), 69.96$ (d, $J$ 12.65Hz, CH), $25.01\left(\mathrm{~d}, J 28.74 \mathrm{~Hz}, \mathrm{CH}_{3}\right) ;{ }^{31} \mathrm{P} \mathrm{NMR}\left(162 \mathrm{MHz}, \mathrm{CDCl}_{3}\right) \delta=147.3$; MS: $m / z(E I)=374([\mathrm{M}]+, 100 \%), 331(80 \%)$; HRMS: calc for $\mathrm{C}_{23} \mathrm{H}_{19} \mathrm{O}_{3} \mathrm{P}: 374.1072$. Found: 374.1079. 


\section{G) Synthesis of the Ru (II) complexes ${ }^{9}$}

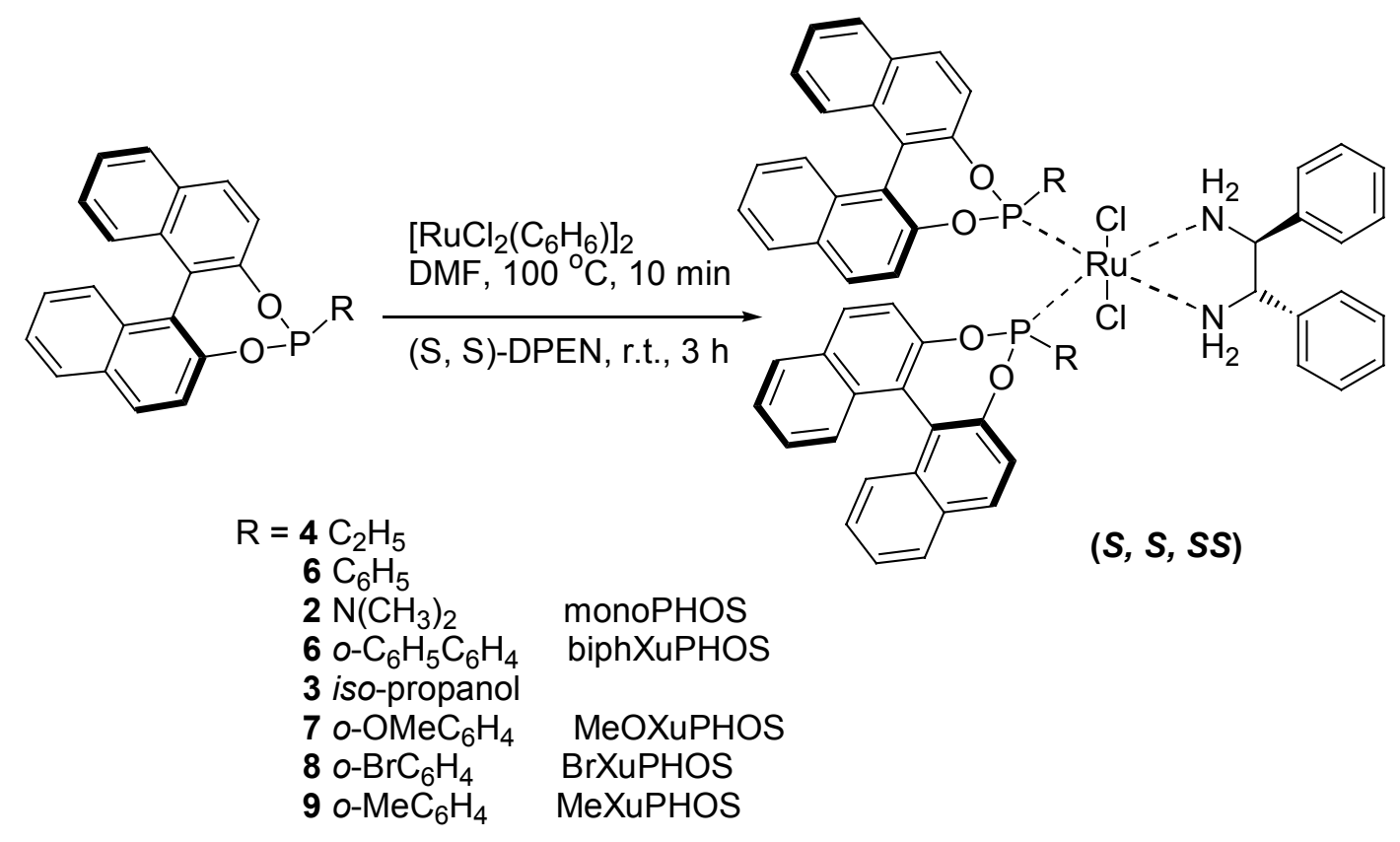

1. Synthesis of $(S, S, S S) \mathrm{Ru}-$ EthylPHOS 4 complex

$\left[\mathrm{RuCl}_{2}\left(\mathrm{C}_{6} \mathrm{H}_{6}\right)\right]_{2}(100 \mathrm{mg}, 0.200 \mathrm{mmol})$ and $(S)$-Et PHOS $4(275.5 \mathrm{mg}, 0.800 \mathrm{mmol}$, 4e.q.) were placed in a 50-ml Schlenk flask. After the air in the flask was replaced with argon, anhydrous DMF (9 ml) was added, the mixture was degassed and stirred under argon at $100{ }^{\circ} \mathrm{C}$ for $15 \mathrm{~min}$ to form a reddish brown solution. After the solution was cooled to room temperature, $(S, S)$-DPEN $(85 \mathrm{mg}, 0.400 \mathrm{mmol}, 2$ e.q.) was added and the mixture was degassed again before it was stirred for $3 \mathrm{~h}$. After the reaction finished, DCM $(30-60 \mathrm{ml})$ was added into the reaction mixture, and at each time it was turned on the high vacuum and back to argon, to do azeotrope to get rid of remaining DMF. The resulting dark brown solid was dried under the high vacuum to give the final product $(S, S, S S) \mathrm{Ru}-\mathrm{Et}$ PHOS (321mg, 75\%). mp $210{ }^{\circ} \mathrm{C}$ (dec.); $[\alpha]_{\mathrm{D}}^{22}=-77.9\left(c 0.1, \mathrm{CH}_{2} \mathrm{Cl}_{2}\right)$; IR: $v_{\max } \mathrm{solid} / \mathrm{cm}^{-1}=2926,1666,1224,945,747,697$; ${ }^{1} \mathrm{H}$ NMR $\left(300 \mathrm{MHz}, \mathrm{CDCl}_{3}\right) \delta=7.95-7.84(6 \mathrm{H}, \mathrm{m}, \mathrm{Ar}-\mathrm{H}), 7.59(4 \mathrm{H}, \mathrm{dd}, J 8.85 \mathrm{~Hz}, J$ $14.89 \mathrm{~Hz}, \mathrm{Ar}-\mathrm{H}), 7.48(2 \mathrm{H}, \mathrm{d}, J$ 8.85Hz, Ar-H), 7.44-7.35 (6H, m, Ar-H), 7.22-7.17 (6H, m, Ar-H), 7.11-7.06 (6H, m, Ar-H), 7.05-7.02 (4H, m, Ar-H), 4.27-4.24 (2H, m, $\left.2 \mathrm{NH}_{2} \mathrm{CH}\right), 4.20-4.15(2 \mathrm{H}, \mathrm{m}, 2 \mathrm{NHH}), 3.73-3.67(2 \mathrm{H}, \mathrm{m}, 2 \mathrm{NHH}), 2.82-2.74(2 \mathrm{H}, \mathrm{m}$, 2PCHH), 2.54-2.40 (2H, m, 2PCHH), 1.00-0.90 (6H, m, 2CH $) ;{ }^{31} \mathrm{P}$ NMR (162 MHz, $\left.\mathrm{CDCl}_{3}\right) \delta=228.8$; LSIMS: $m / z(\mathrm{FAB})=1072\left([\mathrm{M}]^{+}, 100 \%\right), 1037(60 \%), 905(20 \%)$; HRMS: calc for $\mathrm{C}_{58} \mathrm{H}_{50} \mathrm{Cl}^{35}{ }_{2} \mathrm{~N}_{2} \mathrm{O}_{4} \mathrm{P}_{2} \mathrm{Ru}^{102}$ : $1072.1666\left([\mathrm{M}]^{+}\right)$. Found: 1072.1707.

\section{Synthesis of $(S, S, S S) \mathrm{Ru}-\mathrm{PhPHOS} 5$ complex}

$\left[\mathrm{RuCl}_{2}\left(\mathrm{C}_{6} \mathrm{H}_{6}\right)\right]_{2}(100 \mathrm{mg}, 0.200 \mathrm{mmol})$ and $(S)$-PhPHOS $5(314 \mathrm{mg}, 0.800 \mathrm{mmol}$, 4e.q.) were placed in a 50-ml Schlenk flask. After the air in the flask was replaced with argon, anhydrous DMF $(7 \mathrm{ml})$ was added, the mixture was degassed and stirred under argon at $100{ }^{\circ} \mathrm{C}$ for $15 \mathrm{~min}$ to form a reddish brown solution. After the solution was cooled to room temperature, $(S, S)$-DPEN ( $85 \mathrm{mg}, 0.400 \mathrm{mmol}, 2$ e.q.) was added and the mixture was degassed again before it was stirred for $3 \mathrm{~h}$. After the reaction 
finished, DCM $(30-60 \mathrm{ml})$ was added into the reaction mixture, and at each time it was turned on the high vacuum and back to argon, to do azeotrope to get rid of remaining DMF. The resulting dark brown solid was dried under the high vacuum to give the final product $(S, S, S S) \mathrm{Ru}-\mathrm{PhPHOS}(378 \mathrm{mg}, 81 \%)$. m.p. $185^{\circ} \mathrm{C}$ (dec.); $[\mathrm{a}]_{\mathrm{D}}^{22}=-287.8\left(\mathrm{c} 0.1, \mathrm{CH}_{2} \mathrm{Cl}_{2}\right)$; IR: $v_{\max } \mathrm{solid} / \mathrm{cm}^{-1}=3056,2922,1661,1225,945$, 696; ${ }^{1} \mathrm{H}$ NMR $\left(300 \mathrm{MHz}, \mathrm{CDCl}_{3}\right) \delta=8.29(2 \mathrm{H}, \mathrm{d}, J 8.85 \mathrm{~Hz}, \mathrm{Ar}-\mathrm{H}), 8.03-7.58(10 \mathrm{H}$, $\mathrm{m}, \mathrm{Ar}-\mathrm{H}), 7.40-7.11(12 \mathrm{H}, \mathrm{m}, \mathrm{Ar}-\mathrm{H}), 7.07-6.90$ (16H, m, Ar-H), 6.78 (2H, d, J 8.49 $\mathrm{Hz}, \mathrm{Ar}-\mathrm{H}), 6.60(2 \mathrm{H}, \mathrm{d}, J 8.85 \mathrm{~Hz}, \mathrm{Ar}-\mathrm{H}), 4.35-4.32$ (2H, m, 2NH $\left.\mathrm{NH}_{2} \mathrm{CH}\right), 3.75-3.68$ (2H, m, 2NHH), 3.40-3.34 (2H, m, 2NHH); ${ }^{31} \mathrm{P}$ NMR (162 MHz, $\left.\mathrm{CDCl}_{3}\right) \quad \delta=205.7$; MS: $m / z(\mathrm{FAB})=1168\left([\mathrm{M}]^{+}, 100 \%\right), 1133(42 \%) ;$ HRMS: calc for $\mathrm{C}_{66} \mathrm{H}_{50} \mathrm{Cl}^{35}{ }_{2} \mathrm{~N}_{2} \mathrm{O}_{4} \mathrm{P}_{2} \mathrm{Ru}^{102}$ : $1168.1636\left([\mathrm{M}]^{+}\right)$. Found: 1168.1666.

\section{Synthesis of (S, S, SS) Ru-monoPHOS 2 complex}

$\left[\mathrm{RuCl}_{2}\left(\mathrm{C}_{6} \mathrm{H}_{6}\right)\right]_{2}(100 \mathrm{mg}, 0.200 \mathrm{mmol})$ and $(S)$-monophos (287mg, 0.800mmol, 4e.q.) were placed in a 50-ml Schlenk flask. After the air in the flask was replaced with argon, anhydrous DMF $(10 \mathrm{ml})$ was added, the mixture was degassed and stirred under argon at $100{ }^{\circ} \mathrm{C}$ for $10 \mathrm{~min}$ to form a reddish brown solution. After the solution was cooled to $25{ }^{\circ} \mathrm{C},(S, S)$-DPEN $(85 \mathrm{mg}, 0.400 \mathrm{mmol})$ was added and the mixture was degassed again before it was stirred for $3 \mathrm{~h}$. After the reaction finished, the supernatant was removed, and DCM was added in for several times into the reaction mixture, and at each time it was turned on the high vacuum and back to argon. The resulting dark yellow solid was dried under the high vacuum to give $(S)$-monophos, (S, S)-DPEN Ru (II) complex (390mg, 89\%). m.p. $245-247{ }^{\circ} \mathrm{C}$ (dec.); $[\alpha]_{\mathrm{D}}{ }^{20}=-$ 392.7 (c $0.1, \mathrm{CH}_{2} \mathrm{Cl}_{2}$ ); IR: $v_{\max }$ solid $/ \mathrm{cm}^{-1}=2917,2359,1668,1226,944,823 ;{ }^{1} \mathrm{H}$ NMR $\left(300 \mathrm{MHz}, \mathrm{CDCl}_{3}\right) \delta=8.31(2 \mathrm{H}, \mathrm{d}, J 8.85 \mathrm{~Hz}, \mathrm{Ar}-\mathrm{H}), 8.02-7.84(8 \mathrm{H}, \mathrm{m}, \mathrm{Ar}-\mathrm{H})$, 7.47-7.39 (4H, m, Ar-H), 7.34-7.07 (10H, m, Ar-H), 6.94-6.92 (6H, m, Ar-H), 6.73$6.72(4 \mathrm{H}, \mathrm{m}, \mathrm{Ar}-\mathrm{H}), 4.23-4.20$ (2h, m, 2NH $\mathrm{NH}_{2} \mathrm{CH}$, 3.99-3.96 (2H, m, 2NHH), 3.32$3.26(2 \mathrm{H}, \mathrm{m}, 2 \mathrm{NHH}), 2.91\left(12 \mathrm{H}, \mathrm{d}, J 21.85 \mathrm{~Hz}, 2 \mathrm{NMe}_{2}\right) ;{ }^{31} \mathrm{P} \mathrm{NMR}\left(162 \mathrm{MHz}, \mathrm{CDCl}_{3}\right)$ $\delta=172.7$; LSIMS: $m / z(\mathrm{FAB})=1102\left([\mathrm{M}]^{+}, 100 \%\right), 1067(50 \%), 743(30 \%)$; HRMS: calc for $\mathrm{C}_{58} \mathrm{H}_{52} \mathrm{Cl}^{35}{ }_{2} \mathrm{~N}_{4} \mathrm{O}_{4} \mathrm{P}_{2} \mathrm{Ru}^{102}$ : $1102.1884\left([\mathrm{M}]^{+}\right)$. Found 1102.1895.

\section{Synthesis of $(R, R, R R) \mathrm{Ru}$ - biphenyl XuPHOS 6 complex}

$\left[\mathrm{RuCl}_{2}\left(\mathrm{C}_{6} \mathrm{H}_{6}\right)\right]_{2} \quad(79 \mathrm{mg}, \quad 0.158 \mathrm{mmol})$ and $(R)$-biphenyl XuPHOS 6 (297mg, $0.633 \mathrm{mmol}$, 4e.q.) were placed in a 50-ml Schlenk flask. After the air in the flask was replaced with argon, anhydrous DMF $(7 \mathrm{ml})$ was added, the mixture was degassed and stirred under argon at $100{ }^{\circ} \mathrm{C}$ for $15 \mathrm{~min}$ to form a reddish brown solution. After the solution was cooled to room temperature, $(R, R)$-DPEN $(67 \mathrm{mg}, 0.316 \mathrm{mmol}, 2 \mathrm{e} . q$. was added and the mixture was degassed again before it was stirred for $3 \mathrm{~h}$. After the reaction finished, DCM $(30-60 \mathrm{ml})$ was added into the reaction mixture, and at each time it was turned on the high vacuum and back to argon, to do azeotrope to get rid of remaining DMF. The resulting dark brown solid was dried under the high vacuum to give the final product $(R, R, R R) \mathrm{Ru}$-biphenyl XuPHOS $(240 \mathrm{mg}, 58 \%)$. m.p. $210{ }^{\circ} \mathrm{C}$ (dec.); $[\alpha]_{\mathrm{D}}{ }^{19}=+331.8\left(c 0.1, \mathrm{CH}_{2} \mathrm{Cl}_{2}\right)$; IR: $v_{\max } \mathrm{solid} / \mathrm{cm}^{-1}=3049,1668,1225,948$, 747, 695; ${ }^{1} \mathrm{H}$ NMR (300 MHz, $\left.\mathrm{CDCl}_{3}\right) \delta=8.00-7.87(2 \mathrm{H}, \mathrm{m}, \mathrm{Ar}-\mathrm{H}), 7.71-7.66(4 \mathrm{H}, \mathrm{m}$, Ar-H), 7.57-7.50 (6H, m, Ar-H), 7.41-7.34 (4H, m, Ar-H), 7.28-6.93 (24H, m, Ar-H), 6.85-6.66 (10H, m, Ar-H), $6.35(2 \mathrm{H}, \mathrm{d}, J 8.83 \mathrm{~Hz}, \mathrm{Ar}-\mathrm{H}), 4.44-4.42(2 \mathrm{H}, \mathrm{m}$, $\left.2 \mathrm{NH}_{2} \mathrm{CH}\right), 3.84-3.79(2 \mathrm{H}, \mathrm{m}, 2 \mathrm{NHH}), 3.28-3.26(2 \mathrm{H}, \mathrm{m}, 2 \mathrm{NHH}) ;{ }^{31} \mathrm{P}$ NMR $(162$ 
$\left.\mathrm{MHz}, \mathrm{CDCl}_{3}\right) \delta=210.0$; MS: $m / z(\mathrm{EI})=1321\left([\mathrm{M}+\mathrm{H}]^{+}, 100 \%\right), 1037(70 \%)$; HRMS: calc for $\mathrm{C}_{78} \mathrm{H}_{58} \mathrm{Cl}_{2} \mathrm{~N}_{2} \mathrm{O}_{4} \mathrm{P}_{2} \mathrm{Ru}: 1320.2292$. Found 1320.2329.

\section{Synthesis of (S, S, SS) Ru—iso-propyl phosphite 3 complex}

$\left[\mathrm{RuCl}_{2}\left(\mathrm{C}_{6} \mathrm{H}_{6}\right)\right]_{2} \quad(77 \mathrm{mg}, 0.154 \mathrm{mmol})$ and $(S)$-isopropyl phosphite 3 (230mg, $0.616 \mathrm{mmol}$, 4e.q.) were placed in a 50-ml Schlenk flask. After the air in the flask was replaced with argon, anhydrous DMF $(5 \mathrm{ml})$ was added, the mixture was degassed and stirred under argon at $100{ }^{\circ} \mathrm{C}$ for $15 \mathrm{~min}$ to form a reddish brown solution. After the solution was cooled to room temperature, $(S, S)$-DPEN $(65 \mathrm{mg}, 0.307 \mathrm{mmol}, 2$ e.q.) was added and the mixture was degassed again before it was stirred for $3 \mathrm{~h}$. After the reaction finished, DCM $(30-60 \mathrm{ml})$ was added into the reaction mixture, and at each time it was turned on the high vacuum and back to argon, to do azeotrope to get rid of remaining DMF. The resulting dark brown solid was dried under the high vacuum to give the final product $(S, S, S S) \mathrm{Ru}$-isopropyl phosphite $(224 \mathrm{mg}, 64 \%)$. m.p. 160$162{ }^{\circ} \mathrm{C}$ (dec.). $[\alpha]_{\mathrm{D}}{ }^{23}=-306.6\left(c 0.1, \mathrm{CH}_{2} \mathrm{Cl}_{2}\right)$; IR: $v_{\max } \mathrm{solid} / \mathrm{cm}^{-1}=2360,1662,1228$, 942, 807, 695; ${ }^{31} \mathrm{P}$ NMR (162 MHz, $\left.\mathrm{CDCl}_{3}\right) \delta=155.4$; LSIMS: $m / z(\mathrm{FAB})=1132$ $\left([\mathrm{M}]^{+}, 100 \%\right), 1099(30 \%), 935$ (35\%); HRMS: calc for $\mathrm{C}_{60} \mathrm{H}_{54} \mathrm{O}_{6} \mathrm{Cl}^{35}{ }_{2} \mathrm{~N}_{2} \mathrm{P}_{2} \mathrm{Ru}^{102}$ : 1132.1878. Found: 1132.1887.

\section{Synthesis of $(S, S, S S) \mathrm{Ru}-\mathrm{MeO}$ XuPHOS 7 complex}

$\left[\mathrm{RuCl}_{2}\left(\mathrm{C}_{6} \mathrm{H}_{6}\right)\right]_{2}(100 \mathrm{mg}, 0.200 \mathrm{mmol})$ and $(S)-\mathrm{MeO}$ XuPHOS 7 (338mg, 0.800mmol, 4e.q.) were placed in a 50-ml Schlenk flask. After the air in the flask was replaced with argon, anhydrous DMF $(10 \mathrm{ml})$ was added, the mixture was degassed and stirred under argon at $100{ }^{\circ} \mathrm{C}$ for $10 \mathrm{~min}$ to form a reddish brown solution. After the solution was cooled to $25^{\circ} \mathrm{C},(S, S)$-DPEN ( $85 \mathrm{mg}, 0.400 \mathrm{mmol}$ ) was added and the mixture was degassed again before it was stirred for $3 \mathrm{~h}$. After the reaction finished, the supernatant was removed, and DCM was added in for several times into the reaction mixture, and at each time it was turned on the high vacuum and back to argon. The resulting dark yellow solid was dried under the high vacuum to give the metal complex $(S, S, S S) \mathrm{Ru}-\mathrm{MeO}$ XuPHOS (438mg, 89\%). m.p. $270-272{ }^{\circ} \mathrm{C}\left(\mathrm{dec}\right.$.); $[\alpha]_{\mathrm{D}}{ }^{19}$ $=-237.5\left(c 0.02, \mathrm{CH}_{2} \mathrm{Cl}_{2}\right)$; IR: $v_{\max }$ solid $/ \mathrm{cm}^{-1}=3328,2934,1669,1221,945,750 ;{ }^{1} \mathrm{H}$ NMR $(400 \mathrm{MHz}, \mathrm{CDCl} 3): \delta=7.72(2 \mathrm{H}, \mathrm{d}, J 7.95 \mathrm{~Hz}, \mathrm{Ar}-\mathrm{H}), 7.66(2 \mathrm{H}, \mathrm{d}, J 7.91 \mathrm{~Hz}, \mathrm{Ar}-$ $\mathrm{H}), 7.61(2 \mathrm{H}, \mathrm{t}, J 12.00 \mathrm{~Hz}, \operatorname{Ar}-\mathrm{H}), 7.49(2 \mathrm{H}, \mathrm{d}, J 8.83 \mathrm{~Hz}, \mathrm{Ar}-\mathrm{H}), 7.44(2 \mathrm{H}, \mathrm{t}, J$ 8.23Hz, Ar-H), 7.35 (2H, t, J 7.99Hz, Ar-H), 7.28-7.24 (8H, m, Ar-H), 7.16-7.13 (8H, $\mathrm{m}, \mathrm{Ar}-\mathrm{H}), 7.10-7.06$ (6H, m, Ar-H), 7.02-7.00 (6H, m, Ar-H), 6.87 (2H, t, Ar-H), 4.58 $\left(2 \mathrm{H}, \mathrm{m}, 2 \mathrm{NH}_{2} \mathrm{CH}\right), 4.47-4.45(2 \mathrm{H}, \mathrm{m}, 2 \mathrm{NHH}), 4.14-4.10(2 \mathrm{H}, \mathrm{m}, 2 \mathrm{NHH}), 3.97(6 \mathrm{H}, \mathrm{s}$, $\left.2 \mathrm{OCH}_{3}\right) ;{ }^{31} \mathrm{P}$ NMR $\left(162 \mathrm{MHz}, \mathrm{CDCl}_{3}\right) \delta=206.3$. LSIMS: $m / z(\mathrm{FAB})=1228\left([\mathrm{M}]^{+}\right.$, $100 \%), 1193$ (71\%); HRMS: calc for $\mathrm{C}_{68} \mathrm{H}_{54} \mathrm{O}_{6} \mathrm{Cl}^{35}{ }_{2} \mathrm{~N}_{2} \mathrm{P}_{2} \mathrm{Ru}^{102}$ : 1228.188 . Found: 1228.188. (Note: other isotope peaks observed within 10 ppm: 1228.192 (45\%), $1228.190(20 \%), 1228.185(25 \%))$.

\section{Synthesis of $(S, S, S S) \mathrm{Ru}-\mathrm{Br}$ XuPHOS 8 complex}

$\left[\mathrm{RuCl}_{2}\left(\mathrm{C}_{6} \mathrm{H}_{6}\right)\right]_{2}(100 \mathrm{mg}, 0.200 \mathrm{mmol})$ and $(S)$-Br XuPHOS 8 (377mg, 0.800mmol, 4e.q.) were placed in a 50-ml Schlenk flask. After the air in the flask was replaced with argon, anhydrous DMF (10 ml) was added, the mixture was degassed and stirred under argon at $100{ }^{\circ} \mathrm{C}$ for $10 \mathrm{~min}$ to form a reddish brown solution. After the solutin was cooled to $25{ }^{\circ} \mathrm{C},(S, S)$-DPEN $(85 \mathrm{mg}, 0.400 \mathrm{mmol})$ was added and the mixture 
was degassed again before it was stirred for $3 \mathrm{~h}$. After the reaction finished, the supernatant was removed, and DCM was added in for several times into the reaction mixture, and at each time it was turned on the high vacuum and back to argon. The resulting dark yellow solid was dried under the high vacuum and recrystalised in hot DMF $(100 \mathrm{mg} / 3.3 \mathrm{ml})$ at $100^{\circ} \mathrm{C}$ to give the final bright golden crystals $(S, S, S S) \mathrm{Ru}-$ Br XuPHOS (345mg, 65\%). mp $235-237{ }^{\circ} \mathrm{C}$ (dec.); [ a $]_{\mathrm{D}}{ }^{19}=-496.8\left(c 0.1, \mathrm{CH}_{2} \mathrm{Cl}_{2}\right)$; IR: $v_{\max }$ solid $/ \mathrm{cm}^{-1}=2927,2360,1673,1224,954,809 ;{ }^{1} \mathrm{H}$ NMR $\left(300 \mathrm{MHz}, \mathrm{CDCl}_{3}\right)$ $\delta=8.43-8.41(2 \mathrm{H}, \mathrm{m}, \mathrm{Ar}-\mathrm{H}), 8.15-8.14(2 \mathrm{H}, \mathrm{m}, \mathrm{Ar}-\mathrm{H}), 7.92-7.86(4 \mathrm{H}, \mathrm{m}, \mathrm{Ar}-\mathrm{H})$, 7.51-7.26 (6H, m, Ar-H), 7.12-7.10 (10H, m, Ar-H), 6.98-6.84 (12H, m, Ar-H), 6.55$6.30(6 \mathrm{H}, \mathrm{m}, \mathrm{Ar}-\mathrm{H}), 4.55-4.53\left(2 \mathrm{H}, \mathrm{m}, 2 \mathrm{NH}_{2} \mathrm{CH}\right), 4.23-4.20(2 \mathrm{H}, \mathrm{m}, 2 \mathrm{NHH}), 2.88-$ $2.83(2 \mathrm{H}, \mathrm{m}, 2 \mathrm{NHH}) ;{ }^{31} \mathrm{P}$ NMR $\left(162 \mathrm{MHz}, \mathrm{CDCl}_{3}\right) \quad \delta=203.9$; LSIMS: $m / z$ (FAB) $=1291\left([\mathrm{M}-\mathrm{Cl}]^{+}, 100 \%\right), 1247(65 \%), 1196(50 \%), 1079(35 \%)$; HRMS: calc for $\mathrm{C}_{66} \mathrm{H}_{48} \mathrm{Br}^{79} \mathrm{Br}^{81} \mathrm{Cl}^{35}{ }_{2} \mathrm{~N}_{2} \mathrm{O}_{4} \mathrm{P}_{2} \mathrm{Ru}^{102}: 1291.0168\left([\mathrm{M}-\mathrm{Cl}]^{+}\right)$. Found 1291.0167.

\section{Synthesis of $(S, S, S S) \mathrm{Ru}-\mathrm{Me}$ XuPHOS 9 complex}

$\left[\mathrm{RuCl}_{2}\left(\mathrm{C}_{6} \mathrm{H}_{6}\right)\right]_{2}(92 \mathrm{mg}, 0.185 \mathrm{mmol})$ and $(S)$-Me XuPHOS 9 (303 mg, $0.738 \mathrm{mmol}$, 4e.q.) were placed in a 50-ml Schlenk flask. After the air in the flask was replaced with argon, anhydrous DMF (9 ml) was added, the mixture was degassed and stirred under argon at $100{ }^{\circ} \mathrm{C}$ for $10 \mathrm{~min}$ to form a reddish brown solution. After the solutin was cooled to $25^{\circ} \mathrm{C},(S, S)$-DPEN (78 $\mathrm{mg}, 0.370 \mathrm{mmol}$ ) was added and the mixture was degassed again before it was stirred for $3 \mathrm{~h}$. After the reaction finished, the supernatant was removed, and DCM was added in for several times into the reaction mixture, and at each time it was turned on the high vacuum and back to argon. The resulting dark yellow solid was dried under the high vacuum to give the final product $(S, S, S S) \mathrm{Ru}-\mathrm{Me}$ XuPHOS (100 mg, 23\%). mp $250-252{ }^{\circ} \mathrm{C}(\text { dec.); [ a }]_{\mathrm{D}}{ }^{19}=-460$ (c $0.1, \mathrm{CH}_{2} \mathrm{Cl}_{2}$ ); IR: $v_{\max }$ solid $/ \mathrm{cm}^{-1}=3689,2359,1668,1225,947,696 ;{ }^{1} \mathrm{H} \mathrm{NMR}$ $\left(300 \mathrm{MHz}, \mathrm{CDCl}_{3}\right) \delta=8.35(2 \mathrm{H}, \mathrm{d}, J 8.28 \mathrm{~Hz}, \mathrm{Ar}-\mathrm{H}), 8.00(4 \mathrm{H}, \mathrm{d}, J 9.00 \mathrm{~Hz}, \mathrm{Ar}-\mathrm{H})$, 7.87-7.78 (2H, m, Ar-H), 7..73-7.66 (2H, m, Ar-H), $7.45(2 \mathrm{H}, \mathrm{d}, J$ 7.92Hz, Ar-H), 7.34-7.24 (4H, m, Ar-H), 7.12-6.82 (19H, m, Ar-H), 6.61-6.58 (5H, m, Ar-H), 6.376.35 (2H, m, Ar-H), 4.13-4.11 (2H, m, 2NH $\left.\mathrm{NH}_{2} \mathrm{CH}\right), 3.75-3.49$ (2H, m, 2NHH), 2.56$2.48(2 \mathrm{H}, \mathrm{m}, 2 \mathrm{NHH}), 2.22\left(6 \mathrm{H}, \mathrm{s}, 2 \mathrm{Ar}-\mathrm{CH}_{3}\right) ;{ }^{31} \mathrm{P} \mathrm{NMR}\left(162 \mathrm{MHz}, \mathrm{CDCl}_{3}\right) \delta=215.6$; LSIMS: $m / z \quad(\mathrm{FAB})=1196\left([\mathrm{M}]^{+}, 100 \%\right), 1125(30 \%)$; HRMS: calc for $\mathrm{C}_{68} \mathrm{H}_{54} \mathrm{Cl}^{35}{ }_{2} \mathrm{~N}_{2} \mathrm{O}_{4} \mathrm{P}_{2} \mathrm{Ru}^{102}: 1196.1979\left([\mathrm{M}-\mathrm{Cl}]^{+}\right)$. Found 1291.2028.

\section{H) General experimental procedure for the asymmetric hydrogenation catalysed by $(S, S, S S)$ Ru-BrXuPHOS ${ }^{9}$}

In an oven dried round bottom flask $(250 \mathrm{~mL})$, acetophenone $(2.10 \mathrm{~mL}, 2.17 \mathrm{~g}, 18.08$ $\mathrm{mmol})$ and $\left(\mathrm{CH}_{3}\right)_{3} \mathrm{COK}(10 \mathrm{mg}, 0.0904 \mathrm{mmol}, 0.5 \mathrm{~mol} \%)$ were dissolved in dry and degassed 2-propanol (120 mL). (S, S, SS) Ru-BrXuPHOS (12 mg, $0.00904 \mathrm{mmol}$, $0.05 \mathrm{~mol} \%)$ was dissolved in anhydrous and degassed $\mathrm{CH}_{2} \mathrm{Cl}_{2}(6 \mathrm{~mL})$, which was used as the catalyst stock solution and was transferred into the reaction solution above under argon. The mixture was degassed by three vacuum-filling with argon cycles and then it was quickly transferred into the autoclave. It was purged with hydrogen for 10 seconds at 2, 5 and 8 atm respectively, and finally the hydrogen was introduced to 10 $\mathrm{atm}$. The reaction mixture was stirred vigorously at $20-22{ }^{\circ} \mathrm{C}$ for $20 \mathrm{~h}$. The mixture was filtered through a pad of silica gel and the pad was washed with a $50 \%$ solution of ethyl acetate in hexane $(150 \mathrm{~mL})$. The filtrate was concentrated under reduced 
pressure to afford the reduction product. Purification by flash chromatography was applied when appropriate.

\section{I) HPLC, GC analysis and optical rotation data of the reduction products}

1. Data of reduction products (reactions catalyzed by $(\mathrm{S}, \mathrm{S}, \mathrm{SS}) \mathrm{Ru}-\mathrm{BrXuPHOS}$ at room temperature)

(R)-1-phenylethanol: $[\alpha]_{\mathrm{D}}{ }^{22}+45.2\left(c 0.5, \mathrm{CH}_{2} \mathrm{Cl}_{2}\right)\left(\right.$ lit. $^{10}[\alpha]_{\mathrm{D}}{ }^{23}+48.6\left(c 1.0, \mathrm{CH}_{2} \mathrm{Cl}_{2}\right)$, $96 \%$ e.e. $(R)), 90.2 \%$ e.e. $(R)$ by GC (Cyclodextrin- $\beta-236 \mathrm{M}-19$ (CHROMPAC, $50 \mathrm{~m}$ ), $\mathrm{T}=115{ }^{\circ} \mathrm{C}, R$ isomer $19.2 \mathrm{~min}, S$ isomer $\left.20.6 \mathrm{~min}\right)$.

(R)-1-(4'-methylphenyl)ethanol: $[\alpha]_{\mathrm{D}}{ }^{25}+53.2\left(c 0.236, \mathrm{CHCl}_{3}\right)\left(\right.$ lit. $^{11}[\alpha]_{\mathrm{D}}{ }^{22}-54.1(c$ $\left.0.0532, \mathrm{CHCl}_{3}\right), 96 \%$ e.e. $\left.(S)\right), 96.0 \%$ e.e. $(R)$ by $\mathrm{GC}($ Cyclodextrin- $\beta-236 \mathrm{M}-19$ (CHROMPAC, 50m), T $=125^{\circ} \mathrm{C}, R$ isomer $19.6 \mathrm{~min}, S$ isomer $20.7 \mathrm{~min}$ ).

(R)-1-(1'-naphthyl)ethanol: $[\alpha]_{\mathrm{D}}{ }^{21}+74.2(c) 0.3$, ether $)\left(\right.$ lit. $^{9}[\alpha]_{\mathrm{D}}{ }^{25}+77.0(c) 1.02$, ether), $98 \%$ e.e. $(R)), 94.2 \%$ e.e. $(R)$ by GC (Cyclodextrin- $\beta-236 \mathrm{M}-19$ (CHROMPAC, 50m), T $=155^{\circ} \mathrm{C}, S$ isomer $59.6 \mathrm{~min}, R$ isomer $61.7 \mathrm{~min}$ ).

(R)-1-(2'-bromophenyl)ethanol: $[\alpha]_{\mathrm{D}}{ }^{23}+54.9\left(c 0.340, \mathrm{CH}_{2} \mathrm{Cl}_{2}\right)\left(\right.$ lit. $^{9}[\alpha]_{\mathrm{D}}{ }^{24}-60.5(c$ $\left.0.57, \mathrm{CH}_{2} \mathrm{Cl}_{2}\right), 98 \%$ e.e. $\left.(S)\right), 91.0 \%$ e.e. $(R)$ by GC (Cyclodextrin- $\beta-236 \mathrm{M}-19$ (CHROMPAC, 50m), T $=145^{\circ} \mathrm{C}, R$ isomer $24.8 \mathrm{~min}, S$ isomer $29.1 \mathrm{~min}$ ).

(R)-1-(4'-methoxyphenyl)ethanol: $[\alpha]_{\mathrm{D}}{ }^{24}+39.8\left(c 0.300, \mathrm{CHCl}_{3}\right)\left(\right.$ lit. $^{12}[\alpha]_{\mathrm{D}}{ }^{23}-51.9(c$ $\left.1.04, \mathrm{CHCl}_{3}\right), 97 \%$ e.e. $\left.(S)\right), 85.2 \%$ e.e. $(R)$ by GC (Cyclodextrin- $\beta-236 \mathrm{M}-19$ (CHROMPAC, $50 \mathrm{~m}$ ), $\mathrm{T}=130{ }^{\circ} \mathrm{C}, R$ isomer $38.3 \mathrm{~min}, S$ isomer $39.9 \mathrm{~min}$ ).

(R)-1-(4'-bromophenyl)ethanol: $[\alpha]_{\mathrm{D}}{ }^{26}+32.8\left(c\right.$ 1.600, $\left.\mathrm{CHCl}_{3}\right)\left(\right.$ lit. $^{13}[\alpha]_{\mathrm{D}}{ }^{24}-37.6(c$ $\left.1.13, \mathrm{CHCl}_{3}\right), 99 \%$ e.e. $\left.(S)\right), 80.0 \%$ e.e. $(R)$ by GC (Cyclodextrin- $\beta-236 \mathrm{M}-19$ (CHROMPAC, 50m), T $=145^{\circ} \mathrm{C}, R$ isomer $30.5 \mathrm{~min}, S$ isomer $31.9 \mathrm{~min}$ ).

(R)-1-(4'-fluorophenyl)ethanol: $[\alpha]_{\mathrm{D}}{ }^{21}+32.7\left(c 0.880, \mathrm{CH}_{3} \mathrm{OH}\right)\left(\right.$ lit. $^{13}[\alpha]_{\mathrm{D}}{ }^{25}-37.9(c$ $\left.1.0, \mathrm{CH}_{3} \mathrm{OH}\right), 99 \%$ e.e. $\left.(S)\right), 94 \%$ e.e. $(R)$ by $\mathrm{GC}$ (Cyclodextrin- $\beta-236 \mathrm{M}-19$ (CHROMPAC, 50m), T $=120^{\circ} \mathrm{C}, R$ isomer $18.8 \mathrm{~min}, S$ isomer $20.2 \mathrm{~min}$ ).

(R)-1-(2'-naphthyl)ethanol: $[\alpha]_{\mathrm{D}}{ }^{24}+36.3\left(c\right.$ 0.580, EtOH) $\left(\right.$ lit. $^{14}[\alpha]_{\mathrm{D}}{ }^{27}+45.4(c) 1.00$, EtOH), $90 \%$ e.e. $(R)), 85 \%$ e.e. $(R)$ by GC (Cyclodextrin- $\beta-236 \mathrm{M}-19$ (CHROMPAC, $50 \mathrm{~m}), \mathrm{T}=155{ }^{\circ} \mathrm{C}, R$ isomer $57.3 \mathrm{~min}, S$ isomer $\left.58.7 \mathrm{~min}\right)$.

2. Data of reduction products (reactions catalyzed by $(\mathrm{S}, \mathrm{S}, \mathrm{SS}) \mathrm{Ru}-\mathrm{BrXuPHOS}$ in the ice bath)

(R)-1-phenylethanol: $[\alpha]_{\mathrm{D}}{ }^{26}+46.0\left(c 0.84, \mathrm{CH}_{2} \mathrm{Cl}_{2}\right)\left(\right.$ lit. $^{10}[\alpha]_{\mathrm{D}}{ }^{23}+48.6\left(c 1.0, \mathrm{CH}_{2} \mathrm{Cl}_{2}\right)$, $96 \%$ e.e. $(R)), 93.0 \%$ e.e. $(R)$ by GC (Cyclodextrin- $\beta-236 \mathrm{M}-19$ (CHROMPAC, $50 \mathrm{~m}$ ), $\mathrm{T}=115^{\circ} \mathrm{C}, R$ isomer $20.0 \mathrm{~min}, S$ isomer $\left.21.3 \mathrm{~min}\right)$. 
(R)-1-(1'-naphthyl)ethanol: $[\alpha]_{\mathrm{D}}{ }^{28}+77.2\left(c \quad 0.67\right.$, ether) $\left(\right.$ lit. $^{9}[\alpha]_{\mathrm{D}}{ }^{25}+77.0(c) 1.02$, ether), $98 \%$ e.e. $(R)), 99.0 \%$ e.e. $(R)$ by GC (Cyclodextrin- $\beta-236 \mathrm{M}-19$ (CHROMPAC, $50 \mathrm{~m}$ ), $\mathrm{T}=155^{\circ} \mathrm{C}, S$ isomer $60.4 \mathrm{~min}, R$ isomer $62.3 \mathrm{~min}$ ).

(R)-1-(2'-bromophenyl)ethanol: $\left.[\alpha]_{\mathrm{D}}{ }^{25}+54.1(c) 1.18, \mathrm{CHCl}_{3}\right)\left(\right.$ lit. $^{13}[\alpha]_{\mathrm{D}}{ }^{24}-54.6(c$ $\left.1.23, \mathrm{CHCl}_{3}\right), 99 \%$ e.e. $\left.(S)\right), 99.0 \%$ e.e. $(R)$ by GC (Cyclodextrin- $\beta-236 \mathrm{M}-19$ (CHROMPAC, 50m), T $=145^{\circ} \mathrm{C}, R$ isomer $26.2 \mathrm{~min}, S$ isomer $30.7 \mathrm{~min}$ ).

3. Data of reduction products (reactions catalyzed by $(S, S, S S) \mathrm{Ru}-\mathrm{MeOXuPHOS}$ at room temperature)

(R)-1-phenylethanol: $[\alpha]_{\mathrm{D}}{ }^{26}+43.6\left(c\right.$ 0.934, $\left.\mathrm{CH}_{2} \mathrm{Cl}_{2}\right)\left(\right.$ lit. $^{10}[\alpha]_{\mathrm{D}}{ }^{23}+48.6$ (c 1.0, $\left.\mathrm{CH}_{2} \mathrm{Cl}_{2}\right), \quad 96 \%$ e.e. $\left.(R)\right), \quad 88 \%$ e.e. $(R)$ by $\mathrm{GC}$ (Cyclodextrin- $\beta-236 \mathrm{M}-19$ (CHROMPAC, 50m), T $=115^{\circ} \mathrm{C}, R$ isomer $19.2 \mathrm{~min}, S$ isomer $20.5 \mathrm{~min}$ ).

(R)-1-(4'-methylphenyl)ethanol: $[\alpha]_{\mathrm{D}}{ }^{29}+50.2\left(c 0.92, \mathrm{CHCl}_{3}\right)\left(\right.$ lit. $^{11}[\alpha]_{\mathrm{D}}{ }^{22}-54.1(c$ $\left.0.0532, \mathrm{CHCl}_{3}\right), 96 \%$ e.e. $\left.(S)\right), 94 \%$ e.e. $(R)$ by $\mathrm{GC}($ Cyclodextrin- $\beta-236 \mathrm{M}-19$ (CHROMPAC, 50m), T $=125^{\circ} \mathrm{C}, R$ isomer $19.2 \mathrm{~min}, S$ isomer $20.2 \mathrm{~min}$ ).

(R)-1-(1'-naphthyl)ethanol: $[\alpha]_{\mathrm{D}}{ }^{18}+71.8$ (c 0.99 , ether) $\left(\right.$ lit. $^{9}[\alpha]_{\mathrm{D}}{ }^{25}+77.0(c) 1.02$, ether), $98 \%$ e.e. $(R)), \quad 88.0 \%$ e.e. $(R)$ by GC (Cyclodextrin- $\beta-236 \mathrm{M}-19$ (CHROMPAC, 50m), T $=155^{\circ} \mathrm{C}, S$ isomer $59.0 \mathrm{~min}, R$ isomer $61.2 \mathrm{~min}$ ).

(R)-1-(4'-fluorophenyl)ethanol: $[\alpha]_{\mathrm{D}}{ }^{19}+26.1\left(c 0.880, \mathrm{CH}_{3} \mathrm{OH}\right)\left(\right.$ lit. $^{13}[\alpha]_{\mathrm{D}}{ }^{25}-37.9(c$ $\left.1.0, \mathrm{CH}_{3} \mathrm{OH}\right), 99 \%$ e.e. $\left.(S)\right), 89 \%$ e.e. $(R)$ by $\mathrm{GC}$ (Cyclodextrin- $\beta-236 \mathrm{M}-19$ (CHROMPAC, 50m), T $=120^{\circ} \mathrm{C}, R$ isomer $18.9 \mathrm{~min}, S$ isomer $20.4 \mathrm{~min}$ ).

\section{J) References}

1. Roe, A. Organic Reactions, Vol. V, John Wiley and Sons, 1949, 143.

2. Raskina, A. D. Zhurnal. Obshchei. Khimii. 1985, 55, 2262.

3. Drewelies, K.; Latascha, H. P. Angew. Chem. Int. Ed. Engl. 1982, 21, 638.

4. Breeden, S.; Wills, M. J. Org. Chem 1999, 64, 9735.

5. Iriuchijima, S. Synthesis, 1978, 684.

6. Li, Z. M.; Zhou, Z. H.; Wang, L. X.; Zhou, Q.; Tang, C. C. Tetrahedron: Asymmetry, 2002, 13, 145.

7. Reetz, M. T.; Mehler, G. Angew. Chem. 2000, 112, 4047.

8. Reetz, M. T.; Mehler, G. Angew. Chem. Int. Ed. 2000, 39, 3889.

9. Doucet, H.; ohkuma, T.; Murata, K.; Yokozawa, T.; Kozawa, M.; Katayama, E.; England, A. F.; Ikariya, T.; Noyori, R. Angew. Chem. Int. Ed. 1998, 37, 1703. 
10. Hayashi, T.; Matsumoto, Y.; Ito, Y. J. Am. Chem. Soc. 1989, 111, 3426.

11. Carter, M. B.; Schiott, B.; Gutierrez, A.; Buchwald, S. L. J. Am. Chem. Soc. 1994, $116,11667$.

12. Fujii, A.; Hashiguchi, S.; Uematsu, N.; Ikariya, T.; Noyori, R. J. Am. Chem. Soc. 1996, $118,2521$.

13. Nakamura, K.; Matsuda, T. J. Org. Chem. 1998, 63, 8957.

14. Hannedouche, J.; Clarkson, G. J.; Wills, M. J. Am. Chem. Soc. 2004, 126, 986. 
$\mathrm{K}) \mathrm{X}$-ray crystallographic data for $\mathrm{Ru}(\mathrm{II})$ complex derived from $\mathbf{8}$ (local name YJ4ort).

Yj4ort solid state structure

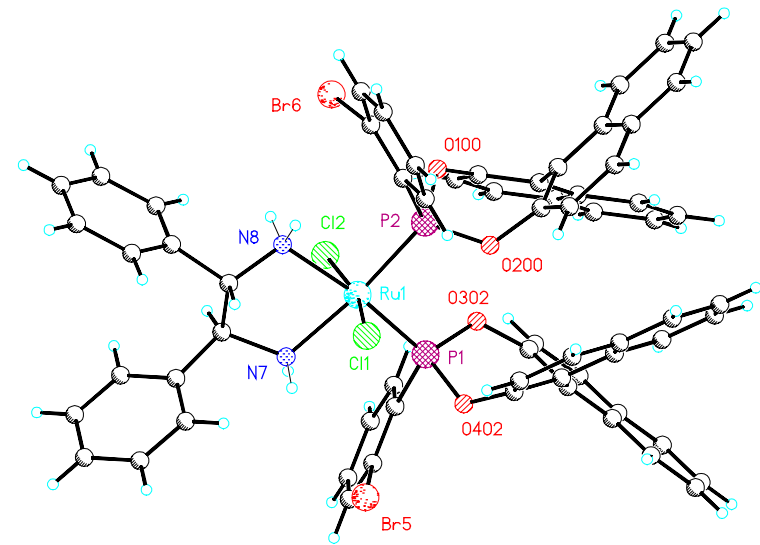

Yj4ort showing internal $\mathrm{H}$ bonds between the phenyl bromide and one of the NHs of each amine (hydrogens not involved in the interaction removed for clarity)

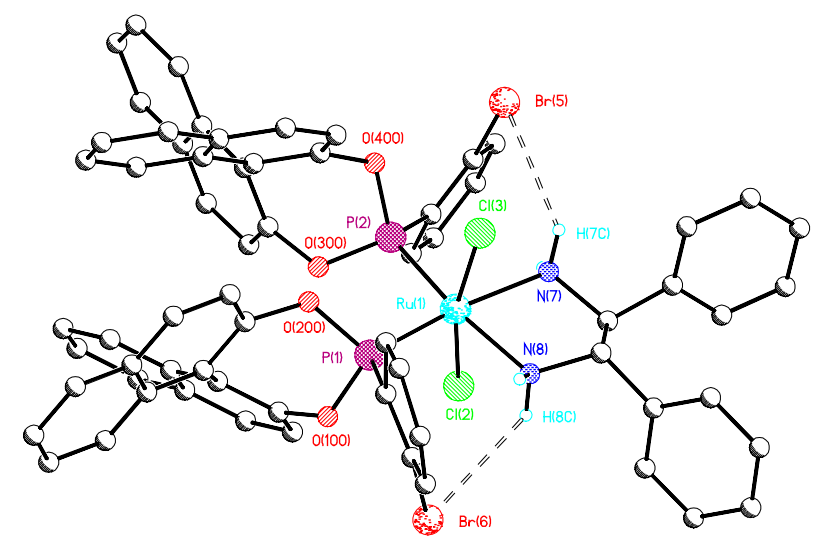

\section{Experimental data for yj4ort}

The assymetric unit contains the complex and some diffuse electron density. This was modelled as disordered solvent, a methanol disordered over two positions (55:45 major minor) and a methanol occupying a similar position as a molecule of water. (45:55 methanol to water).

The torsion angle along the Ru-P1-C501-C502(Br5)bond is -46.36 ( 0.74) Ru1 - P1 C501 - C502

The torsion angle along the Ru-P1-C601-C602(Br6) bond is -39.69 ( 1.55) Ru1 - P2 C601 - C602 
There are short contacts between some of the NHs of the amine ligand and the bromine of the bromophenyl group. These are tabulated below Specified hydrogen bonds (with esds except fixed and riding $\mathrm{H}$ )
$\mathrm{D}-\mathrm{H}$
H...A
D...A
$<($ DHA $)$
0.92
N7-H7C...Br5
0.92
3.05
$3.615(15)$
121.1
N8-H8C...Br6

\section{Crystal Data}

C67.50 H54.50 Br2 Cl2 N2 O6 P2 Ru, M=1383.36, Monoclinic, space group $\mathrm{C} 2(1)$ (c-unique)

$\mathrm{a}=16.649(2), \mathrm{b}=19.163(2), \mathrm{c}=27.224(3) \mathrm{A}$,

alpha $=90$ deg., beta $=90$ deg., gamma $=89.58$ deg.,

$\mathrm{U}=8685.0(19) \mathrm{A}^{\wedge} 3$ (by least squares refinement on 8192 reflection positions),

$\mathrm{T}=180(2) \mathrm{K}$, lambda $=0.71073 \mathrm{~A}, \mathrm{Z}=4$,

$\mathrm{D}(\mathrm{cal})=1.058 \mathrm{Mg} / \mathrm{m}^{\wedge} 3, \mathrm{~F}(000)=2798$.

$\mathrm{mu}($ MoK-alpha $)=1.237 \mathrm{~mm}^{\wedge}-1$.

Crystal character: yellow block.

Crystal dimensions $0.32 \times 0.28 \times 0.12 \mathrm{~mm}$,

\section{Data Collection and Processing.}

Siemens SMART (Siemens, 1994) three-circle system with CCD area detector.

The crystal was held at 180(2)

$\mathrm{K}$ with the Oxford Cryosystem Cryostream Cooler (Cosier \& Glazer, 1986).

Maximum theta was $29.07 \mathrm{deg}$.

The hkl ranges were $-12 / 21,-26 / 25,-35 / 36$.

27051 reflections measured, 18346 unique $[\mathrm{R}$ (int) $=0.1274]$.

Absorption correction by Semi-empirical from equivalents;

minimum and maximum transmission factors: $0.36 ; 0.91$.

No crystal decay

\section{Structure Analysis and Refinement.}

The unit cell metric suggested the possibility of a C-centred orthorhombic lattice, rather than the apparent monoclinic lattice and systematic absences suggested that the space group might be C222(1). The structure could only be solved with monoclinic symmetry and space group C112(1) was used to allow ready comparison with the possible orthorhombic lattice. After refinement, it became clear that the asymmetric unit did not contain any additional symmetry, but there was extensive pseudoorthorhombic twinning (twin factor $0.432(2)$ ). Only with refinement of this twinning parameter could the R-value be reduced below about 0.2 .

The structure was solved by direct methods

using SHELXS (Sheldrick, 1990) (TREF) with additional light atoms found by Fourier methods. Disordered solvent atoms were interpreted as one $\mathrm{MeOH}$ molecule disordered over two positions and one sharing a site with a water molecule.

Hydrogen atoms were added at calculated positions and refined using a riding 
model; $\mathrm{H}$ atoms were not found for the solvent molecules. Anisotropic displacement parameters were used for all non-H atoms;

$\mathrm{H}$-atoms were given isotropic displacement parameters equal to 1.2 times the equivalent isotropic displacement parameter

of the atom to which the $\mathrm{H}$-atom is attached.

The expected chirality of the ligands was confirmed by the value of the Flack parameter.

Absolute structure parameter $\mathrm{x}=0.00(2)$.

Floating origin constraints were generated automatically.

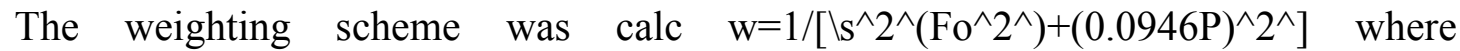
$\mathrm{P}=\left(\mathrm{Fo}^{\wedge} 2^{\wedge}+2 \mathrm{Fc}^{\wedge} 2^{\wedge}\right) / 3$.

Goodness-of-fit on $\mathrm{F}^{\wedge} 2$ was 0.850 ,

$\mathrm{R} 1[$ for 6829 reflections with

$\mathrm{I}>2 \operatorname{sigma}(\mathrm{I})]=0.0962, \mathrm{wR} 2=0.2447$.

Data / restraints / parameters 18346/ 583/ 647.

Extinction coefficient $0.00128(14)$.

Largest difference Fourier peak and hole 1.312 and -0.811 e. $\mathrm{A}^{\wedge}-3$.

The relatively large final $\mathrm{R}$-value was not unexpected because of the extensive twinning, not

all of which could be compensated for through refinement of the twin factor, in view of the

significant deviation of gamma from 90 deg which must have led to imprecise overlap of

the reflections with their twin equivalents.

Refinement used SHELXTL (Sheldrick, 1997).

We thank EPSRC and Siemens Analytical Instruments for grants in support of the diffractometer.

Additional material available from the Cambridge Crystallographic Data Centre comprises $\mathrm{H}$-atom coordinates, thermal parameters and the remaining bond lengths and angles.

References

[ALCOCK, N.W. \& MARKS, P.J. (1994), J. Appl. Cryst. 27, 200-200.]

COSIER, J. \& GLAZER, A. M. (1986), J. Appl. Cryst. 19, 105-107.

SHELDRICK, G.M. (1990), Acta Cryst. A46, 467-473

SHELDRICK, G.M. (1993), Acta Cryst. D49, 18-23

SHELDRICK,G.M.(1997), SHELXTL Ver. 5.1, Bruker Analytical X-ray Systems, Madison, Wis. USA.

SIEMENS (1994), SMART User's manual, Siemens Industrial Automation Inc, Madison, Wis. USA. 
L) 1H-NMR spectra for organic compounds lacking CHN analysis data.

\section{Compound $\mathbf{8}$}

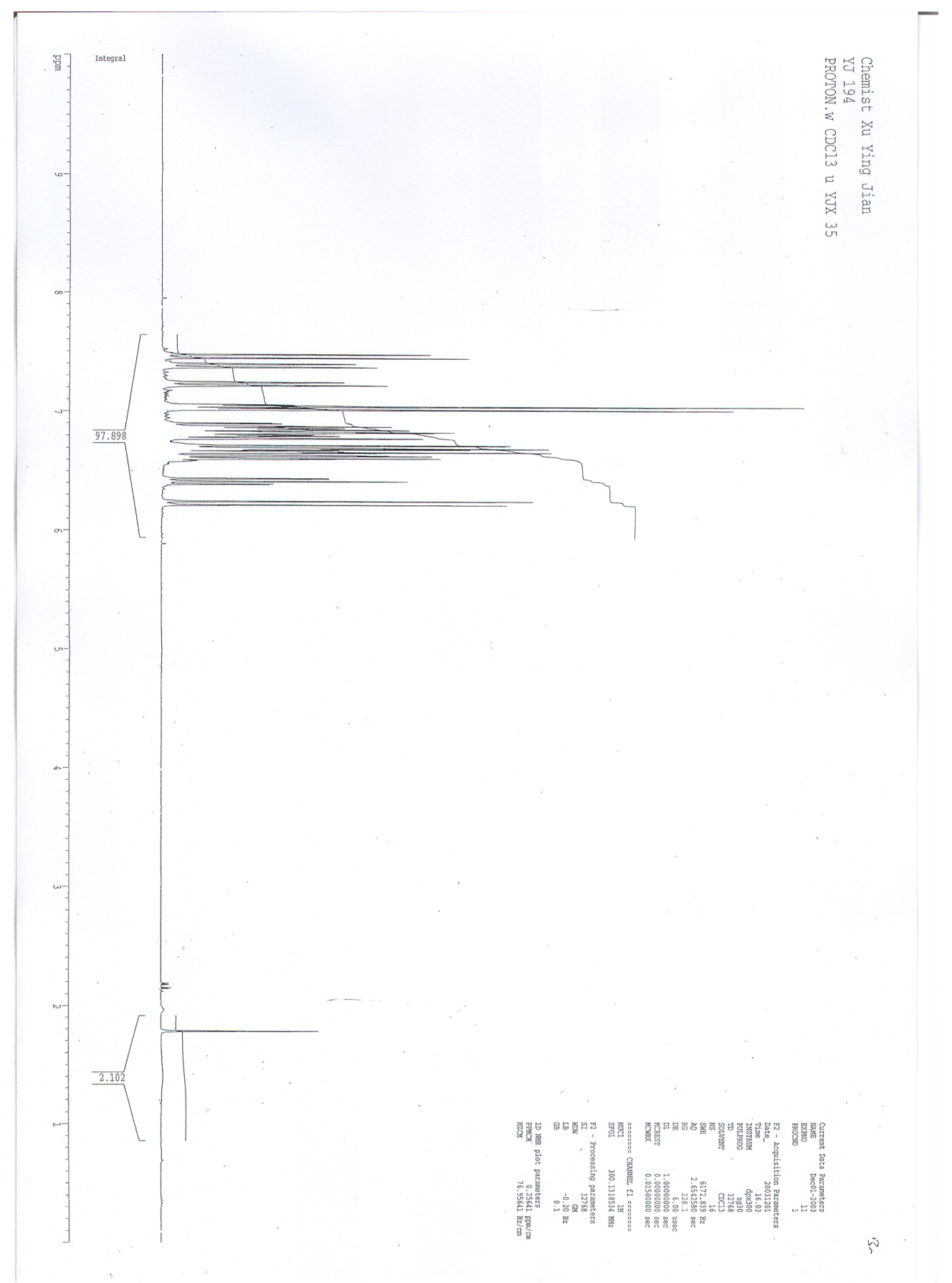


Compound 6

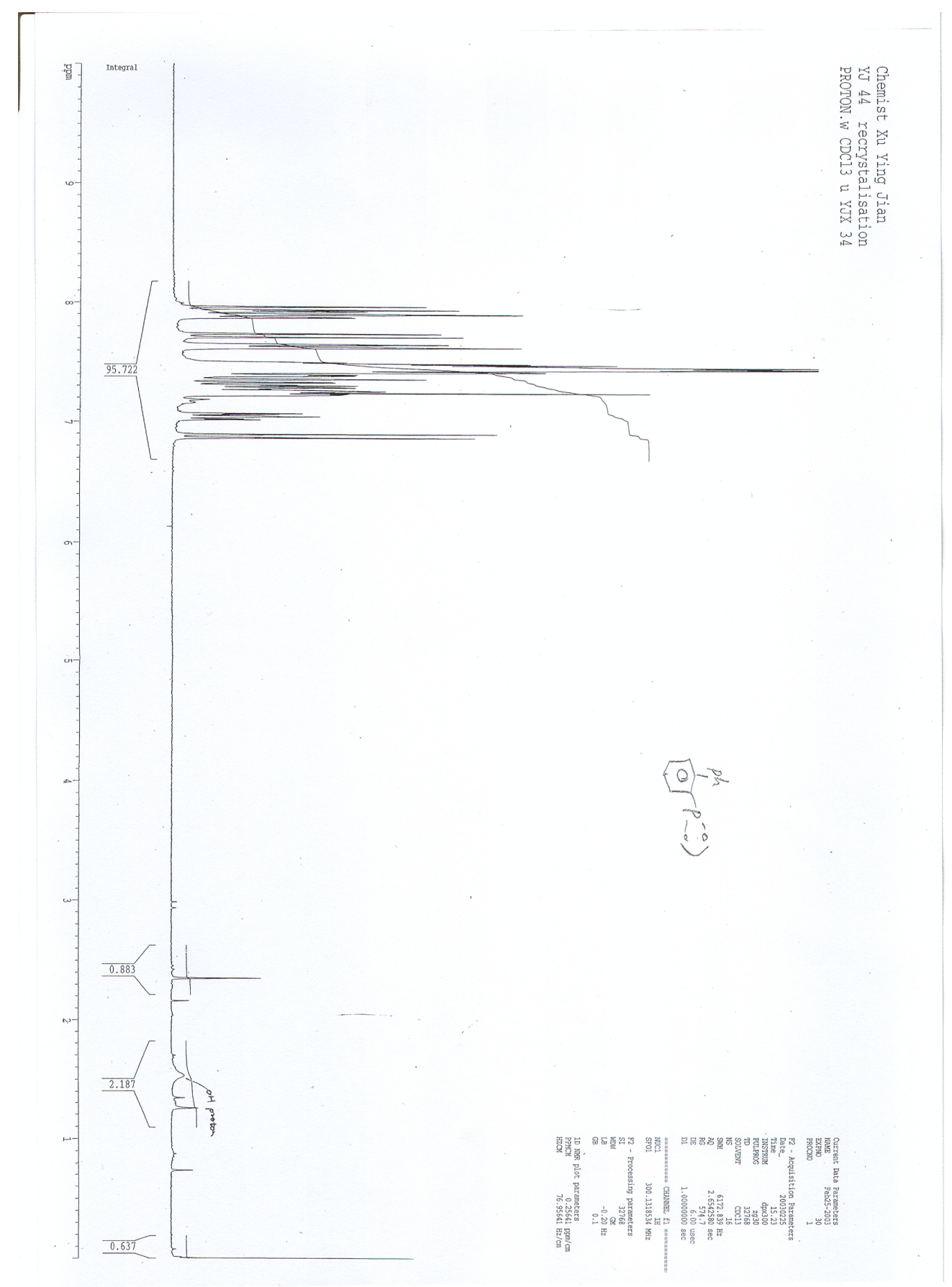


Compound 9

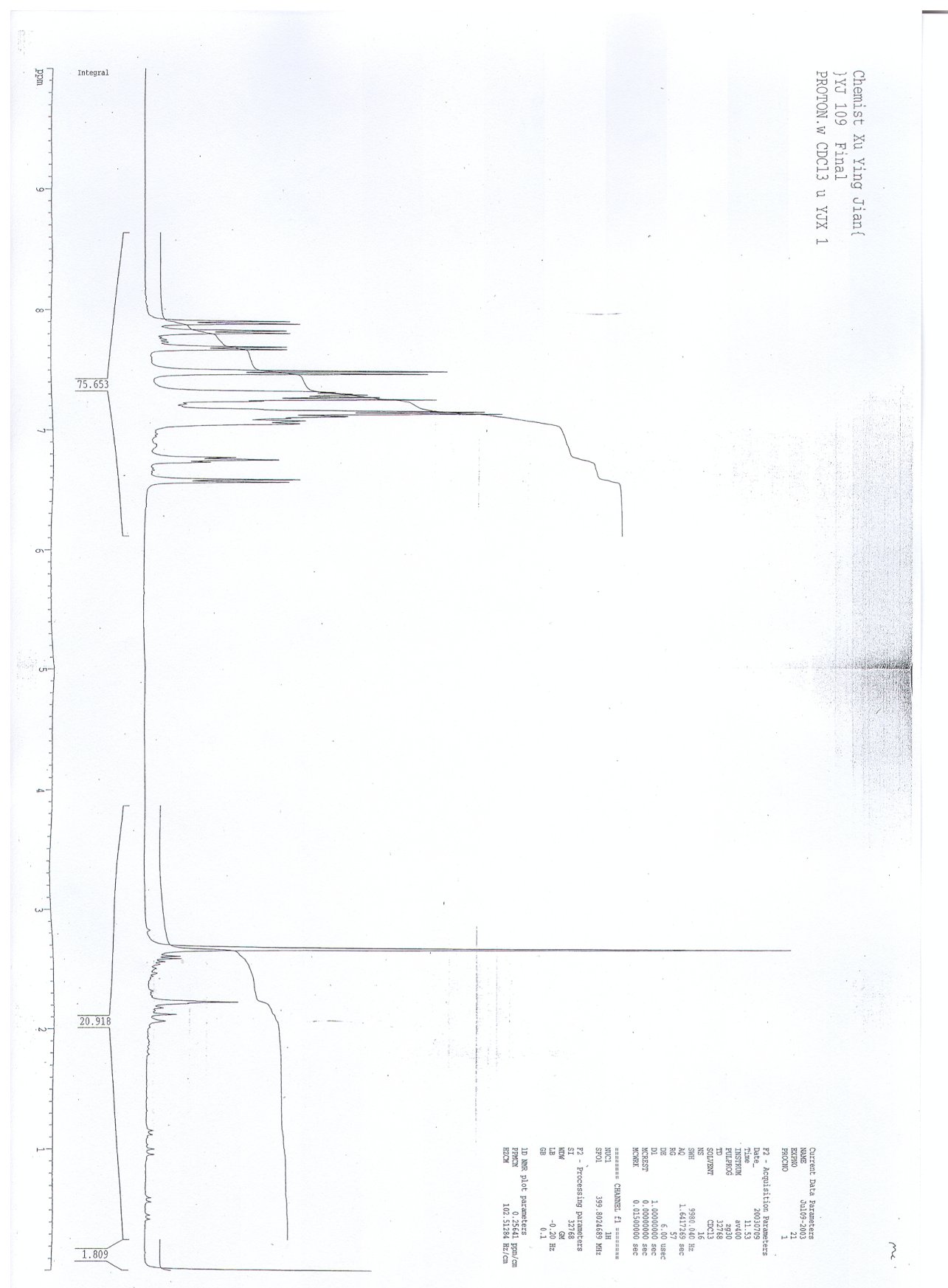

